\section{D) Check for updates}

Cite this: Mater. Adv., 2020 1,2592

Received 13th August 2020, Accepted 3rd September 2020

DOI: 10.1039/d0ma00599a

rsc.li/materials-advances

\title{
Bioinspired materials for water-harvesting: focusing on microstructure designs and the improvement of sustainability
}

\author{
Fan Zhang ${ }^{\mathrm{ab}}$ and Zhiguang Guo (D) *ab
}

\begin{abstract}
In recent years, environmental pollution issues have gradually increased the cost of obtaining clean water for humans. Inspired by natural creatures, collecting water from the atmosphere has become a hot research topic. Herein, a theoretical model regarding the transportation behavior of water droplets and the waterharvesting mechanism of three classic organisms are summarized. Then, the microstructure design methods of the water-collecting surface are discussed, such as the one-dimensional structure inspired by spider silk and cactus, the two-dimensional structure inspired by Namib desert beetles, and the composite structure inspired by multiple creatures. In addition to improving the collection efficiency through microstructure design, the sustainability of the water-harvesting materials is also crucial, which is rarely reported. It is meaningful to provide references on how to improve the mechanical stability, chemical stability, and antibacterial properties. Finally, conclusions are drawn and some prospects for the future development of bionic water-collecting materials are proposed.
\end{abstract}

\section{Introduction}

In the natural and socio-economic system, the shortage of fresh water has always restricted the rapid progress and development of mankind. ${ }^{1-7}$ Since the 21 st century, global climate change, population growth, river siltation, large-scale water intake, and rising pollution levels have placed multiple pressures on water resources, aquatic organisms, and terrestrial ecosystems worldwide. ${ }^{8-12}$ With the excessive growth of population and the continuous enhancement of the greenhouse effect, fewer fresh water resources can be used directly by humans. ${ }^{13-15}$ Globally, freshwater mainly comes from glaciers, lakes, rainfall, groundwater, etc. ${ }^{16}$ Although the total amount is sufficient, due to the imbalance of time and geographical distribution, the distribution of freshwater in different regions is vastly different, resulting in serious water shortages in some parts of the world. According to statistics, $71 \%$ (4.3 billion people) of the world's population, such as in India, Egypt, and Saudi Arabia, is facing moderate or even severe water shortages. ${ }^{17}$ Therefore, obtaining more clean fresh water resources has become a hot topic that needs to be urgently solved today.

\footnotetext{
${ }^{a}$ Hubei Collaborative Innovation Centre for Advanced Organic Chemical Materials, Ministry of Education, Key Laboratory for the Green Preparation and Application of Functional Materials, Hubei University, Wuhan 430062, People's Republic of China. E-mail: zguo@licp.cas.cn; Fax: +86-931-8277088; Tel: +86-931-4968105

${ }^{b}$ State Key Laboratory of Solid Lubrication, Lanzhou Institute of Chemical Physics, Chinese Academy of Sciences, Lanzhou 730000, People's Republic of China
}

In recent decades, seawater desalination technology has been widely used in freshwater collection in arid areas. ${ }^{18-22}$ However, due to the high cost and geographical limitations, it cannot be widely promoted. It has forced people to develop a new way of collecting water to address the supply of clean drinking water for billions of people. Fog is a kind of water resource present in the air and its total amount accounts for about $10 \%$ of the storage capacity of all freshwater lakes on Earth. ${ }^{23}$ Collecting water from fog can greatly alleviate the water shortage problem for those living in arid areas. Due to the evaporation of oceans, rivers, and lakes, the air is rich in water vapor, which flows to arid areas and encounters cold air that condenses into tiny droplets. ${ }^{24}$ Scientists have found that in nature, there are many organisms that can effectively trap the tiny droplets in the air to survive in arid regions, ${ }^{25-28}$ such as the Namib desert beetle living in desert areas. ${ }^{29}$ The back of this creature has a unique wettable structure and morphology, consisting of alternating patterns of hydrophobic shell and hydrophilic bump, which helps to capture moisture from the air containing fog droplets to meet its daily survival needs. Inspired by nature, the research on bioinspired materials came into being and became a hot topic. ${ }^{30-42}$ In the past ten years, it has made great achievements. ${ }^{43}$ According to the previous literature, by imitating the micro-nano structure, morphology, and wettability of organisms such as spider silk, cactus, and desert beetles, a variety of design and preparation methods of bioinspired water collection materials have been produced. ${ }^{44-46}$ Exploring and imitating the water collection mechanism of 
different organisms are the best ways to design high-efficiency bioinspired water collection surfaces. However, sustainability is a key factor that restricts their development. ${ }^{47,48}$ The existing water catchment surface is extremely fragile and cannot withstand the harsh natural environment. Due to the long-term humid environment, the safety performance of the collected water also needs to be evaluated. Therefore, it is necessary to discuss how to extend the service life and improve the safety performance of the water collection surface.

In this review, the background and historical development of fog collection are first outlined. In the second part, the theoretical model regarding the transportation behaviour of water droplets and the water-harvesting mechanism of three classic organisms are summarized. Then, according to the different mechanisms, different microstructure design methods are classified, including one-dimensional structure, two-dimensional structure, and composite structure. In addition to improving the water collection efficiency through the structural design, we have also paid special attention to the sustainability of the water collection surface. The methods for improving the mechanical stability, chemical stability, and antibacterial properties are discussed for the first time. Finally, the conclusions of this review are drawn and the future development of biomimetic water collection materials is put forward.

\section{Basic theories and natural organisms in water harvesting}

Water harvesting is the collection and use of small water droplets floating in the air. The process can be roughly divided into three parts, namely, the capture, transportation, and storage of water droplets. The capture and transportation process of water droplets is strongly related to the surface wettability, which is mainly determined by the surface chemical composition and surface roughness. Many theoretical models related to surface wettability have been developed, including Young's contact equation $^{49}$ (Fig. 1a), Wenzel model $^{50}$ (Fig. 1b), and Cassie model $^{51}$ (Fig. 1c). They explain how roughness affects the wetting state of water droplets on solid surfaces. Surface wettability plays a decisive role in the capture process of droplets. When the solid surface is hydrophilic, the droplets are in the Wenzel state and are easier to adhere to the solid surface. However, due to the pinning effect, it needs to overcome greater resistance to fall off the surface. On the contrary, liquid droplets in the Cassie state are relatively difficult to adhere but help to continuously refresh the mist-collection surface. In the process of droplet transport, studies have found that small water droplets captured on the biological surface always spontaneously move from one end to the other, which is called the directional movement of water droplets, which is exactly the key to the water harvesting process. There are two basic theoretical models, namely, surface energy gradients and Laplace pressures, which explain the water harvesting mechanism of directional droplet transport.

\subsection{Basic theories}

2.1.1. Surface energy gradient law. Surface wettability is a key factor in controlling the contact state between water droplets and solids. ${ }^{52,53}$ The apparent surface energy of the hydrophilic area is always higher than the apparent surface energy of the hydrophobic area and the liquid always tends to move from the low surface energy area to the high surface energy area. The law of surface energy gradient is as follows (Fig. 1d). ${ }^{54}$

$$
F=\int_{L_{1}}^{L_{2}} \gamma\left(\cos \theta_{a}-\cos \theta_{r}\right) \mathrm{d} l
$$

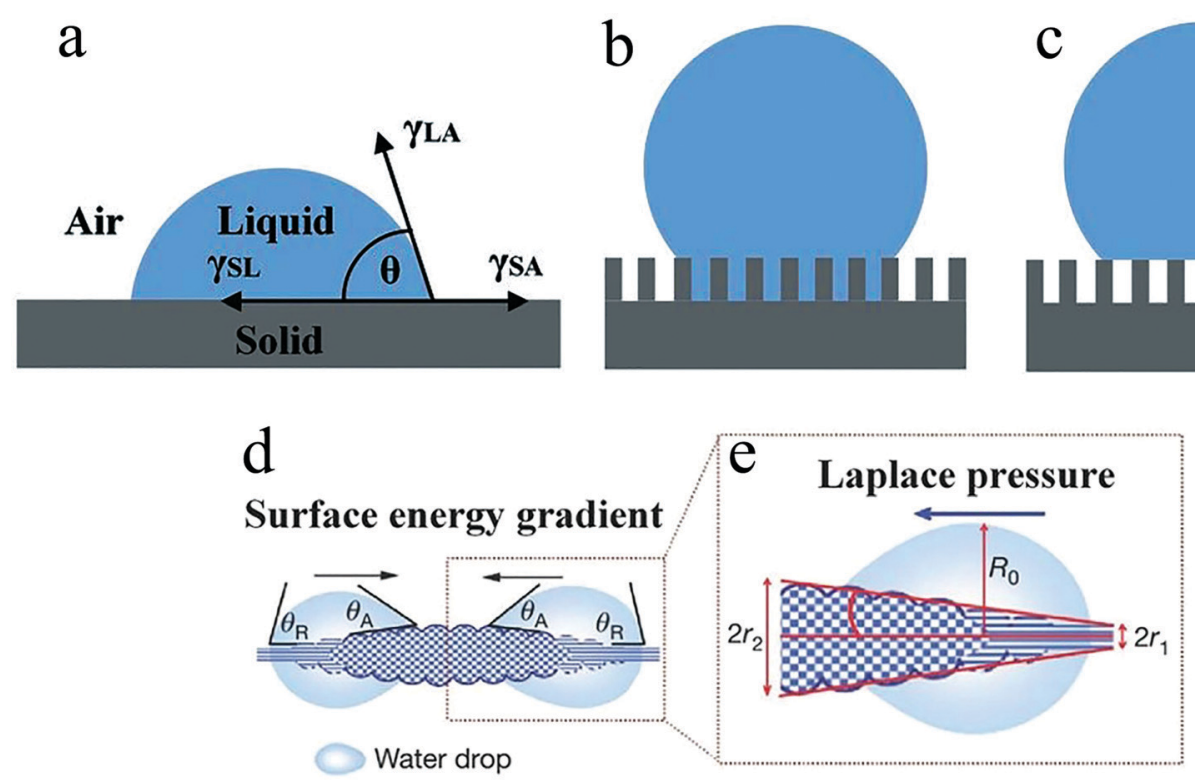

Fig. 1 Different wetting behaviours of the solid surface: (a) Young's model, (b) Wenzel's model, (c) Cassie's model, (d) surface energy gradient, (e) Laplace pressure. Reproduced with permission. ${ }^{25}$ Copyright 2010, Nature. 
where $\gamma$ is the surface tension of water, $\cos \theta_{\mathrm{a}}$ and $\cos \theta_{\mathrm{r}}$ are the forward and backward contact angles of water, respectively, and $\mathrm{d} l$ is an integral variable along the length of the low surface energy region (hydrophobic region L1) to the high surface energy region (hydrophilic region L2). When the contact angles at both ends of the droplet are inconsistent, the difference in the contact angle drives the droplets to move from the high contact angle area to the low contact angle area. It should be emphasized that the difference in the contact angle is only the appearance and the energy gradient is the essence.

2.1.2. Laplace pressure equation. After observing the water collection process of natural organisms, scientists found that the asymmetrical geometric shapes at both the ends can promote the directional movement of water droplets. The Laplace pressure equation is as follows (Fig. 1e). ${ }^{55}$

$$
\Delta P=\int_{r 1}^{r 2} \frac{2 \gamma}{\left(r+R_{0}\right)^{2}} \sin \beta \mathrm{d} z
$$

Here, $r$ is the local radius of the spindle knot or cone, $R_{0}$ is the radius of the droplet, $\gamma$ is the surface tension of water, $\beta$ is the half-apex angle of the spindle knot or cone bottom, and $z$ is the integral variable from the tip to the base. The Laplace pressure of the water drop at the low curvature part (base, at r2) is smaller than the Laplace pressure of the high curvature part (tip, at $\mathrm{r} 1$ ). The water droplets move spontaneously from the tip to the bottom, driven by the Laplace pressure difference.

The combination of surface energy gradient and Laplace pressure is the main driving force for the directional movement of water droplets in the fog collection process, which helps the droplets to transform from the captured state to a usable state without additional energy input, thus greatly speeding up the fog collection efficiency.

\subsection{Natural organisms}

2.2.1. Namib desert beetle. In nature, many organisms have the ability to collect water from air. ${ }^{56,57}$ In 2001, Parker and Lawrence discovered a species of beetle that lives in the Namib Desert. ${ }^{29}$ This beetle collects drinking water from winds containing fog. When the beetle collects water, it tilts its body forward, spreads its wings, and exposes the dorsal shell with many bumps to the wind (Fig. 1a). After chemical tests, it was proved that these uneven surfaces are composed of a waxy region of a hydrophilic portion and a non-waxy region of a hydrophobic portion (Fig. 1b and c). The diameter of the small water droplets in the air is about $1-40 \mathrm{~mm},{ }^{24}$ which are captured by the hydrophilic bumps on the back of the desert beetle under the action of wind. Then, lots of water droplets gather and grow until they cover the entire bump. When the ratio of the mass of the water drop to the surface contact area increases rapidly, the capillary action is not enough to support the weight of the water droplet. Under the effect of gravity of the water droplet, it departs from the hydrophilic protrusion and rolls onto the back hydrophobic channel, and continues along the hydrophobic channel, sliding down and falling into the mouth of the desert beetle.
The temperature of the Namib Desert is close to $40{ }^{\circ} \mathrm{C}$ in sunlight. At this temperature, the water droplets on the back of the desert beetle are successfully collected instead of being vaporized, which proves that the rate of collecting water on the back of the beetle is greater than the rate of gasification. It shows that the strategy of preparing bioinspired water collection materials by imitating the alternate wettability model on the back of the desert beetle is successful. Nowadays, more and more researchers are focusing on alternating wetting surfaces and have produced many artificial water-collecting materials based on desert beetles. ${ }^{58-60}$

2.2.2. Spider silk. Spider silk has excellent mechanical and chemical properties. Every morning, the surface of spider silk is stained with water droplets and distributed evenly, which has inspired people to develop new water collection materials. In 2010, Zheng et $a .^{25}$ completely revealed the mechanism of water collection on the spider silk surface by analysing the surface morphology (Fig. 2d-f). The puff with a certain periodicity is separated by two main axes, which are composed of spider silk nanofibrils. These nanofibrils exhibit a high degree of hydrophilicity, which are conducive for capturing small water droplets in the air. The hydrophilic puff gradually bulges with the increase in the mass of water droplets, forming a periodic spindle knot (Fig. 2d), which is an important reason for the moistened spider silk to be able to collect water directionally. Subsequently, the main function of the spindle knot is no longer to condense water but to collect water and the joints become the main condensation point. The enlarged images of the spindle knot and joint images are shown in Fig. 2e and f, respectively, showing highly random nanofibrils. The water droplets condensed on the joint spontaneously move to the spindle knot under the action of surface energy gradient and Laplace pressure. Water collected in this way cannot eventually be collected in the same place but is evenly distributed on the spindle knot of the spider silk, causing the entire spider web to get wet.

2.2.3. Cactus. The desert cactus lives in arid areas that lack groundwater (Fig. 2g), which survives on its unique water collection function. In 2012 , Ju et al. $^{45}$ revealed a new way of natural water collection by observing cactus in the Chihuahua Desert. The enlarged view of the cactus under a scanning electron microscope shows that its structure is mainly divided into three parts, namely, the barbed tip, the spine with gradual grooves, and the trichomes (Fig. 2h). The water droplets condense and grow at the tips of the spines and barbs, then move to the bottom under the influence of Laplace pressure, and are finally absorbed by the hydrophilic trichomes. There are many gradual grooves on the spine of the cactus (Fig. $2 \mathrm{i}$ and $\mathrm{j}$ ), which cause the surface free energy to gradually increase from the tip to the bottom of the spine. It is another directional driving force on the surface of the spine. The water droplets collected at the bottom of the barb (Fig. 2k) move directionally from the spine tip to the bottom under the combination of surface energy gradient and Laplace pressure. The highly hydrophilic trichomes quickly absorb and store water droplets from the spine. It promotes the continuous transportation of water 

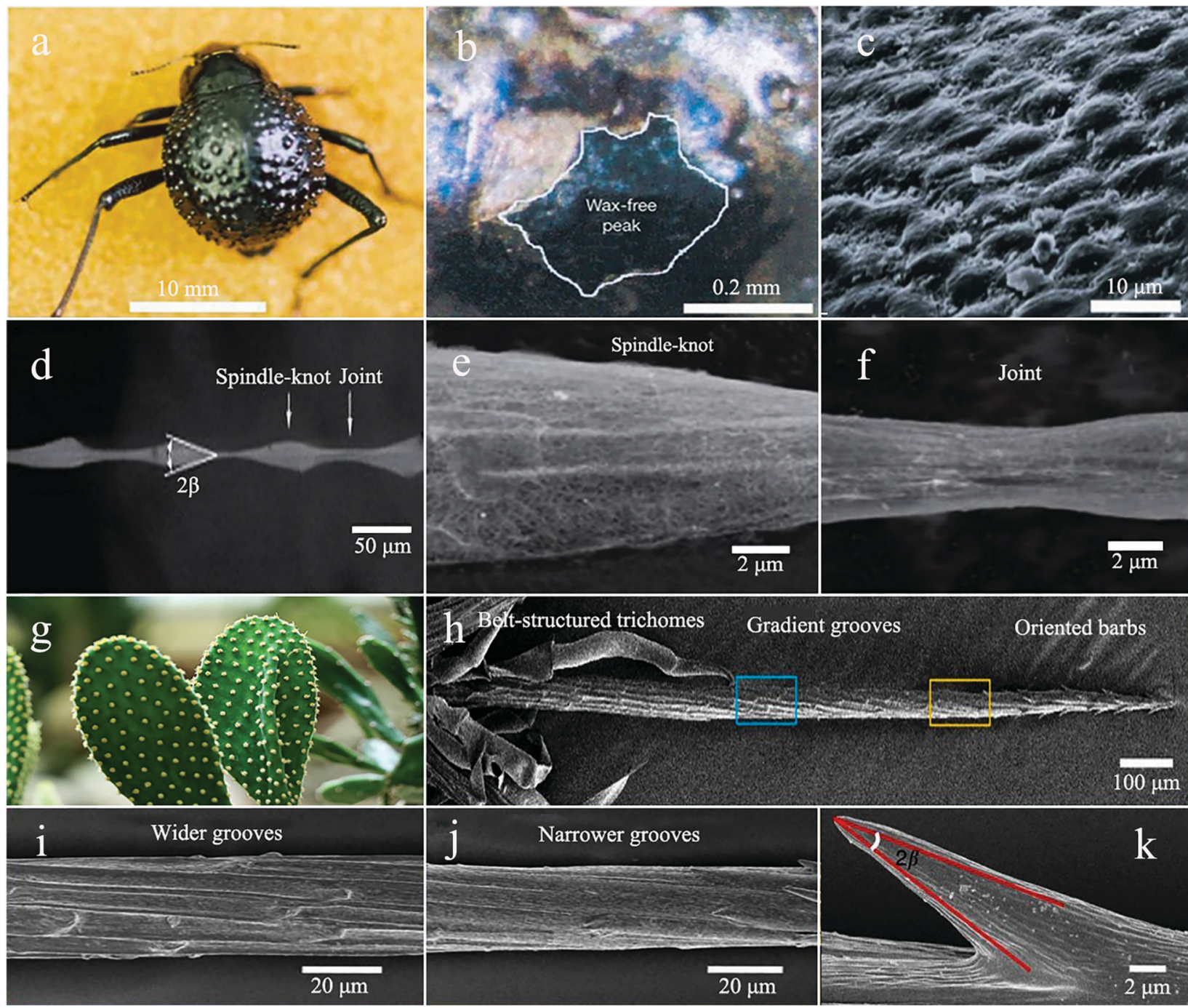

Fig. 2 (a) Image of the back of the Namib Desert Beetle. (b) SEM image of the depressed areas are marked by the staining (waxy, colored), while the peaks of the bumps remain unstained (wax-free, black). (c) The SEM image of the textured surface of the depressed areas. Reproduced with permission. ${ }^{29}$ Copyright 2001, Nature. (d) The periodic spindle-knots linking with the joints. (e and f) Enlarged image of the spindle knots and joints. Reproduced with permission. ${ }^{25}$ Copyright 2010, Nature. (g) The optical image of cactus. (h) The SEM image of the spine divided into three regions, the tip with oriented barbs, the middle with gradient grooves, and the bottom with belt-structured trichomes. (i and j) Gradient grooves. (k) The barbs. Reproduced with permission. ${ }^{45}$ Copyright 2012, Nature Communications.

droplets and reduces evaporation. The unique asymmetric structure and gradient wettability of the cactus spines are important reasons for the efficient water collection ability of the cactus, which provides inspiration to researchers for preparing water collection materials.

\section{The microstructure designs}

Inspired by nature, bioinspired materials are widely used in the field of water harvesting. For example, by imitating the microstructure of spider silk, cactus spines, and desert beetles, the corresponding bionic one-dimensional surface, two-dimensional surface, and three-dimensional composite structure are designed. Here, we briefly review some strategies for designing biomimetic water collection materials.

\subsection{Bionic one-dimensional surface}

3.1.1. Bionic fibers. The periodic spindle knot possessed by moist spider silk is a key factor in its ability to successfully collect water. By imitating spider silk, scientists successfully prepared a series of bioinspired artificial spider silk (BAS). ${ }^{36,61,62}$ Bai et al. ${ }^{63}$ used the dip coating method to produce a series of BASs with spindle junctions, in which the chemical composition and surface nanostructure were cleverly designed. When nylon fiber was immersed in the polymer solution and pulled out horizontally, due to Rayleigh instability, a string of polymer droplets fell on the fiber and became spindle knots after drying (Fig. 3a). Bai first produced fibers with rough PMMA (intrinsic contact angle of $68.4^{\circ}$ ) spindle junctions and smooth PMMA joints. It was observed that tiny water droplets randomly coagulated and were driven towards the spindle knot 


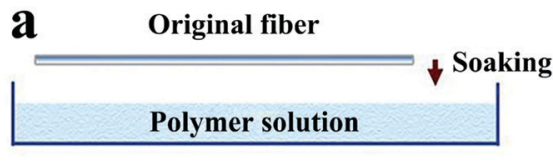

$\downarrow$ Draw fiber out quickly

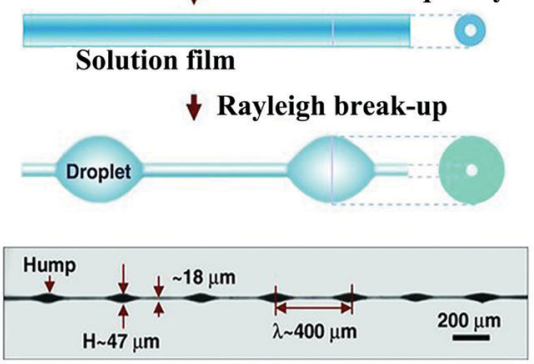

c
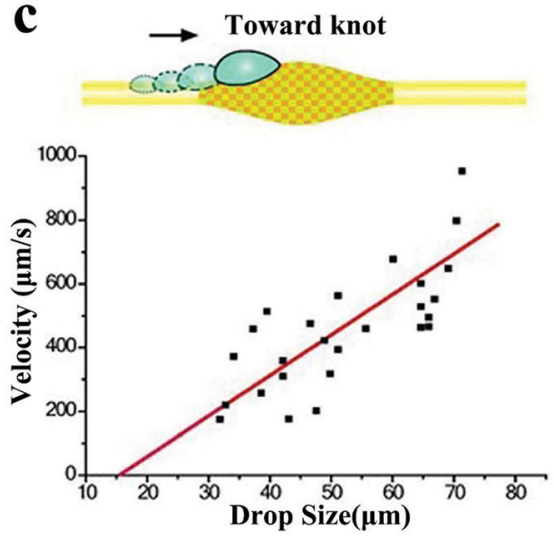
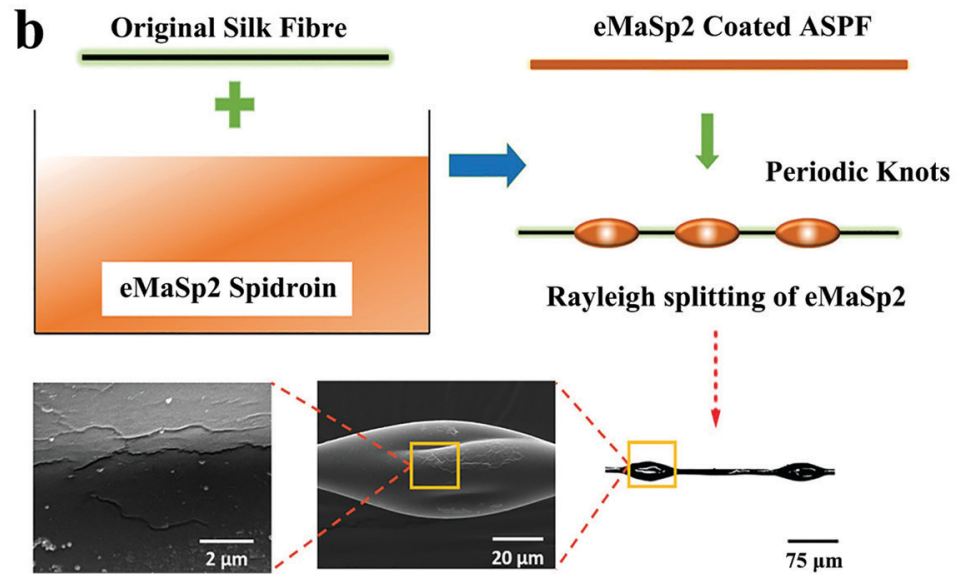

Rayleigh splitting of eMaSp2

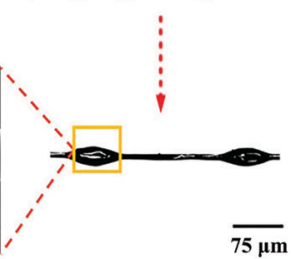

d

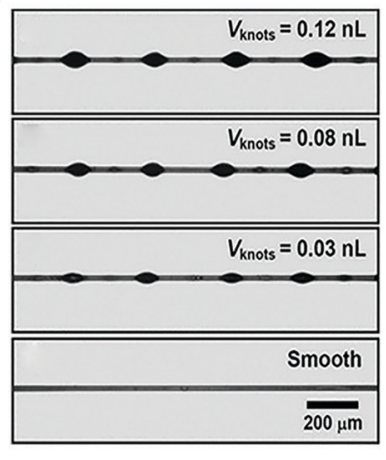

e

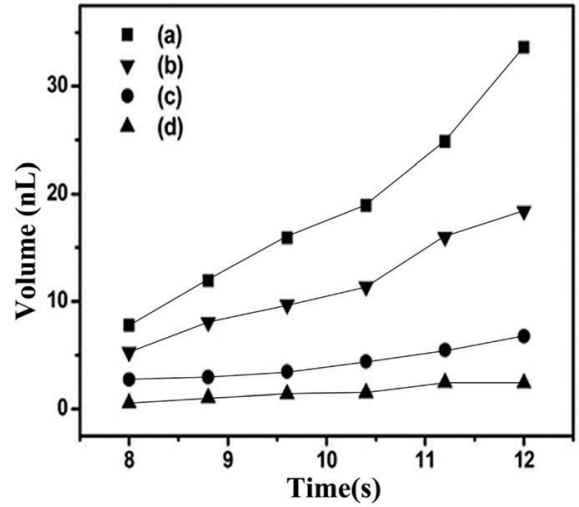

Fig. 3 (a) Artificial fibre manufacturing process with spindle structure. Reproduced with permission. ${ }^{67}$ Copyright 2011, Wiley-VCH. (b) The preparation method of ASPF. Reproduced with permission. ${ }^{70}$ Copyright 2020, Wiley-VCH. (c) Relationship between the droplet size and velocity on the fibres with rough PMMA spindle knots. Reproduced with permission. ${ }^{63}$ Copyright 2010 , Wiley-VCH. (d and e) Relationship between the size of the spindle knot on the BAS and the volume of water collected. Reproduced with permission. ${ }^{64}$ Copyright 2011, Wiley-VCH.

until larger droplets were combined. Then, the same method was used to produce hydrophobic rough PS spindle knots. Unexpectedly, the droplets generated on the hydrophilic PMMA fibers were also driven towards the hydrophobic PS spindle knots. In order to eliminate the effect of roughness, the fibers were treated in a solvent atmosphere and the movement of water droplets on the smooth hydrophobic PS spindle knots and hydrophilic PMMA spindle knots were observed. The results showed that the water droplets were still driven towards the spindle knots. At the same time, they also proved that the size of the droplet was proportional to its moving velocity (Fig. 3c). This work compared the force of surface energy gradient and Laplace pressure. The water droplets overcame the force of surface energy gradient and moved from hydrophilic area to hydrophobic area under the action of Laplace pressure. But the data seemed worthy of further discussion. The Laplace pressure was controlled by the size and curvature of the spindle knots, and the force of the surface energy gradient was controlled by the difference in the water contact angle between the spindle knots and fibers. These factors should be used as single variables to observe the motion state of water droplets on the bionic fibers so as to compare the strength of surface energy gradient and Laplace pressure.

In addition, in order to study the effect of spindle knot geometry on the water collection capacity, $\mathrm{Bai}^{64}$ used different solution viscosities, solution surface tensions, and fiber extraction speeds to produce four different sizes of BAS. All BASs had the same length and contained four main shaft knots, which were at a similar distance from each other (Fig. 3d). These BASs were placed under the same conditions for the water collection test and it was found that BAS with large spindle knots would collect more water than BAS with small spindle knots (Fig. 3e). Hou et $a .^{65}$ further studied the effect of the periodicity of spindle knots on the suspension performance. They found that BAS had a higher water collection capacity than ordinary fibers so that the collected tiny droplets were quickly transported to form larger water droplets at a given location. In addition, due to the greater length of the three-phase contact line (TCL), the BAS with two spindle knots had greater suspension capacity than the BAS with a single spindle knot. Huang et al. ${ }^{66}$ proposed new models of capillary adhesion based on the roughness and curvature characteristics of the wetted spider silk spindle knots, which was conducive for the adhesion of larger pearl-like water 
droplets. The force of capillary adhesion could be improved by changing the roughness and curvature of the spindle knot. In addition, in the work of Tian, Wang, Chen, Hou et al., ${ }^{65,67-69}$ different coating polymers, fiber fineness, hump diameter, and distance between the knots was studied to determine the water collection performance. They found that increasing the fiber diameter, spindle knot size, and periodicity all extended the length of TCL, resulting in higher volume thresholds to promote greater water droplet aggregation.

In a recent study, Hu et al. ${ }^{70}$ used an all silk protein fiber (ASPF) with periodic knots as the main fiber of BAS. ASPF was lighter than synthetic polymer and the corresponding water collection performance reached the highest level than the bionic fibers reported before. The degummed silk fibers were highly water-absorbent. After surface modification, the formed spindle knots helped them to collect water directionally. During the preparation process, the fibers were immersed horizontally in the eMaSp2 Spidroin solution with a concentration of $10 \%$ and then pulled out steadily, which formed a film (Fig. 3b). Due to Rayleigh instability, the film split into tiny droplets distributed along the fiber. It was placed in a controlled environment for methanol vapor treatment and the ASPF with eMaSp2 bionic spindle knots was obtained. Significant surface energy gradient was produced between the eMaSp2 knots with porous surface and fibers, which helped to enhance the stability of TCL. In the water collection test, compared with the maximum volume threshold of $2.2 \mu \mathrm{L}$ reached by the degummed silk fibers at $250 \mathrm{~s}$, ASPF reached $6.6 \mu \mathrm{L}$ at $690 \mathrm{~s}$, which was three times that of degummed silk fibers. The water collection efficiency was expressed by the volume mass index (VTMI) of the fiber mass and the ASPF was found to be 100 times higher than the highest reported water collection efficiency. The weight of the PVDF-coated nylon fibers were 252 times than $\mathrm{ASPF}^{44}$ but it could only collect twice the volume of water $(13.1 \mu \mathrm{L})$. It is worth mentioning that the water collection efficiency of ASPF is relatively high in the initial stage but it cannot achieve a continuous spontaneous process. After reaching the maximum water collection capacity, the water droplets hang on spindle knots and can only be removed slowly by gravity, which affects the subsequent water collection process.

Bionic fiber is a one-dimensional water-collecting material that is easy to prepare. By optimizing the curvature of the spindles on the fibers, chemical gradients, and roughness gradients, tiny water droplets can be captured and pushed in a controlled direction. When the amount of water reaches saturation, the strength of the fibers is also a factor that has to be considered. However, the arrangement of the spindles on the bionic fibers is relatively scattered and the collected water droplets are distributed on the entire fiber, making it difficult to obtain continuous water flows. The cactus thorn with conical structure is another highly efficient one-dimensional water collection material, which inspired new design ideas to achieve continuous water collection.

3.1.2. Conical structure. The unique structure of the cactus (such as the spines with gradient grooves and barbs at the tips) and the coordination between the spines and the trichomes are the keys to its successful water collection. ${ }^{45}$ The droplets deposit on the barbs and spine tips, gather under Laplace pressure, and move towards the middle of the spine. There is a gradient groove in the middle of the spine, which causes the water droplets to be simultaneously subjected to the force generated by the surface energy gradient along the ridge. These two forces together drive the water droplets to move directionally towards the trichomes. In order to achieve the same water collection function, scientists have prepared a series of cactus-inspired conical structures. ${ }^{71-73}$ Cao et al. $^{74}$ have developed an improved organic solvent-free magnetic particle-assisted molding (MPAM) technology that can mass produce cactus-like microtips in a short period of time. The array of hydrophobic conical tips composed of PDMS and MP, together with the hydrophilic cotton fabric, constitutes a mist collection system. The tiny droplets are deposited on the conical tip and move to the hydrophilic bottom under the action of Laplace pressure. During the atomization process, the fusion of tiny droplets will release their surface energy, which is also conducive for the movement of the water droplets on the hydrophobic surface. Heng et al. ${ }^{75}$ prepared a $\mathrm{ZnO}$ wire with many branches to simulate the directional micro barbs on the spine of the cactus. All wires have a conical shape and their diameter gradually increases from the tip to the root. In the water collection test, the average amount of water collected by the artificial structure is 1.4-5.0 times that of the cactus, showing good water collection efficiency.

Ju et al. ${ }^{76}$ manufactured a conical copper wire (CCW) with gradient wettability through gradient chemical etching and subsequent gradient chemical modification. By comparing the movement of the droplets on the hydrophilic CCW and the hydrophobic CCW, it was found that the CCW with gradient wettability has a higher droplet collection rate. For hydrophilic surfaces, droplets have a faster transport rate. But after removing the previously collected water droplets, the surface is easily covered by a liquid film. Compared with the dry surface, the water droplets in the mist flow are more likely to rebound when colliding with the liquid film, which results in a slow droplet growth rate. ${ }^{77}$ In contrast, the hydrophobic surface has a faster droplet growth rate and a slow transport rate (Fig. 4c). Gradient wettability CCW has a gradually decreasing wettability from the base to the tip, which makes it possible to combine the above two advantages to create an efficient water collection device. In the follow-up work, in order to study the impact of the arrangement of the cone array on the water collection efficiency, Ju made PDMS cone arrays with different arrangements (Fig. 4b). ${ }^{78}$ Among them, the hexagonal cone array has a larger deposition area and tiny water droplets will not only deposit on the windward side of the cone but also on the upwind side (Fig. 4a), which makes it more efficient than the quadrilateral cone array (Fig. 4d). These works discussed in detail the influence of the wettability of the conical structure on the water collection capacity. The conical structure with gradient wettability gave the water droplets extra driving force and accelerated the transportation speed, which was a huge improvement and provided a new direction for future defogging tasks. 

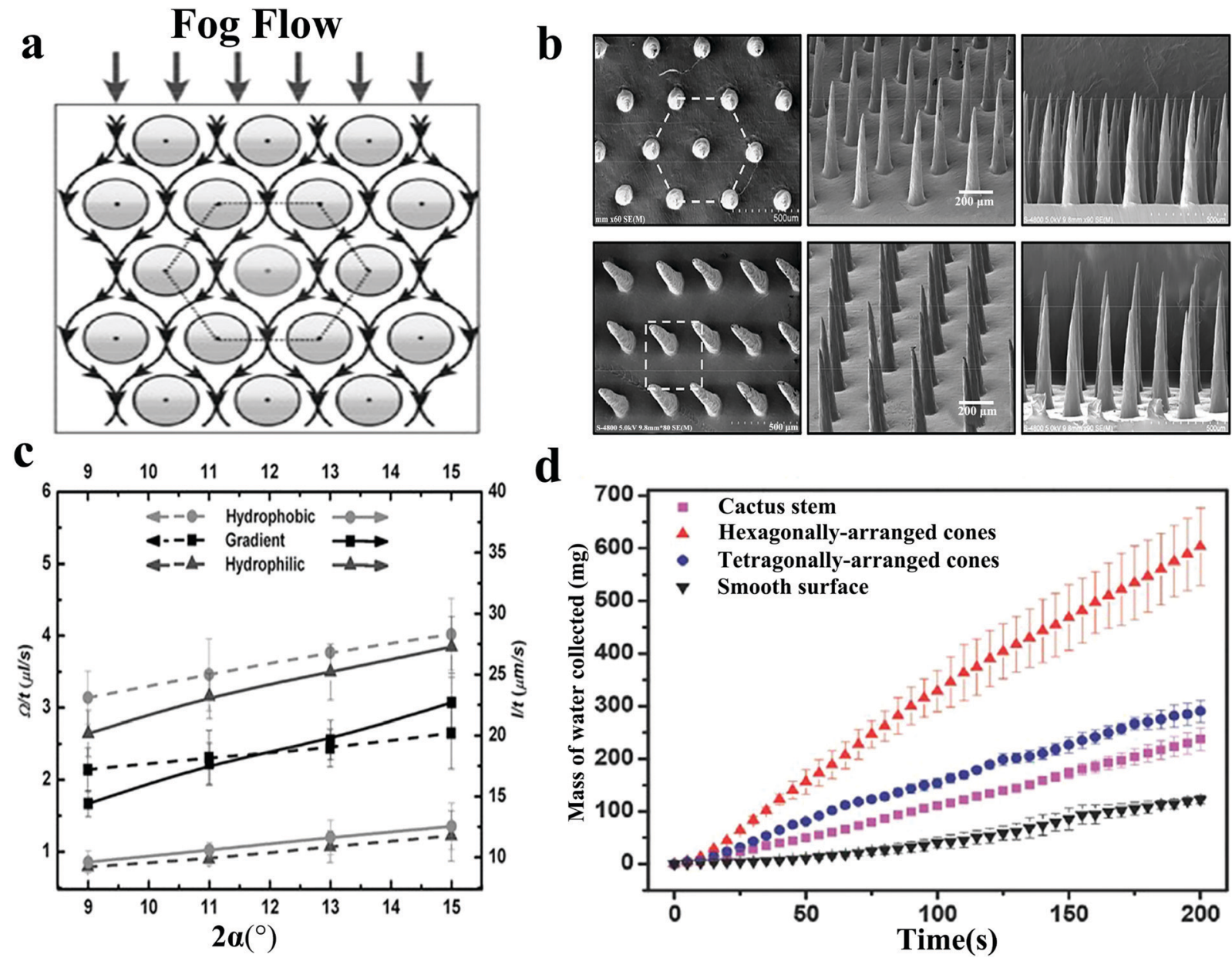

d

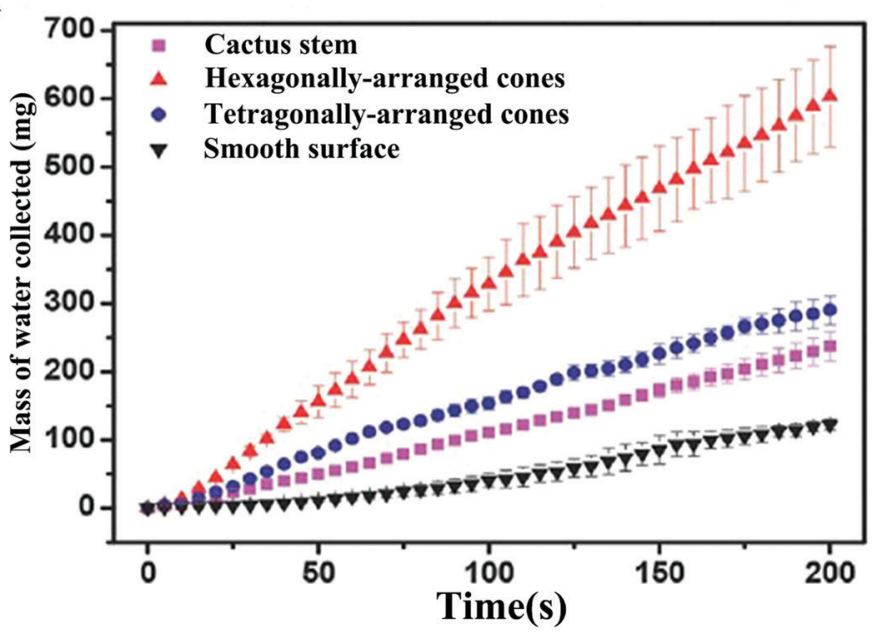

Fig. 4 (a) Fog flow route around the conical array with hexagonal arrangement. (b) Hexagonal and quadrilateral PDMS conical arrays. Reproduced with permission. ${ }^{78}$ Copyright 2014, Wiley-VCH. (c) The effect of the apex angles of three different CCWs on the fog collection behaviour. The dotted and solid lines represent the droplet growth rate and movement rate, respectively. Reproduced with permission. ${ }^{76}$ Copyright 2013 , Wiley-VCH. (d) Under the same conditions, the comparison of the mass of water collected on four different surfaces. The surface with hexagonally-arranged cones shape has the highest fog collection efficiency. Reproduced with permission. ${ }^{78}$ Copyright 2014, Wiley-VCH.

In fact, manufacturing a controllable multi-spine array is challenging and traditional methods are difficult to meet the requirements for preparing high-performance fog collection materials. In recent research, based on 3D printing technology, Li et al. ${ }^{79}$ manufactured a water collector with miniature bionic branch spines and controllable wettability, and developed composite materials to post-process it, resulting in excellent water collection performance. By observing the performance of the spine at different tip angles $\left(10^{\circ}, 20^{\circ}, 30^{\circ}, 40^{\circ}, 50^{\circ}\right)$ during the water collection process, it was found that the spiny spine with a sharp angle of $10^{\circ}$ had the highest water collection efficiency (Fig. 5c). At the same time, the superhydrophobic nanomaterials were sputtered on the surface and it was found that the water-collecting efficiency of the cactus-simulated thorn with a superhydrophobic coating is much higher than that of the 3D printed cactus-simulated thorn without a coating. The reason is that the directional transport of the collected water droplets on the superhydrophobic nano-coating is beneficial for refresh the water droplets at the deposition site, thereby achieving continuous movement. After studying the number and arrangement of the branch clusters of the cactussimulated spiny array, it was found that the surface with four branch cluster arrays had the best water collection efficiency after rotating and moving (Fig. 5a, b, d). This design, based on 3D printing technology, has high efficiency and low cost, and the device can be easily expanded for large-scale water collection in the future. Benefitting from chemical corrosion or $3 \mathrm{D}$ printing technology, bioinspired materials with conical structures have been manufactured by a fog-harvesting method similar to that of the cactus, which is attributed to the surface energy gradients and Laplace pressure. Among them, metal wires and polymers are both optional materials. However, these conical shapes are easy to break. The water collection performance of the equipment will be greatly reduced as the surface coating falls. In the future preparation processes, attention should be paid to maintaining the mechanical durability and chemical durability.

Compared with the bionic fiber, the preparation process of the bionic cone structure is more complicated. However, it can realize the continuity of the water collection process. The water 

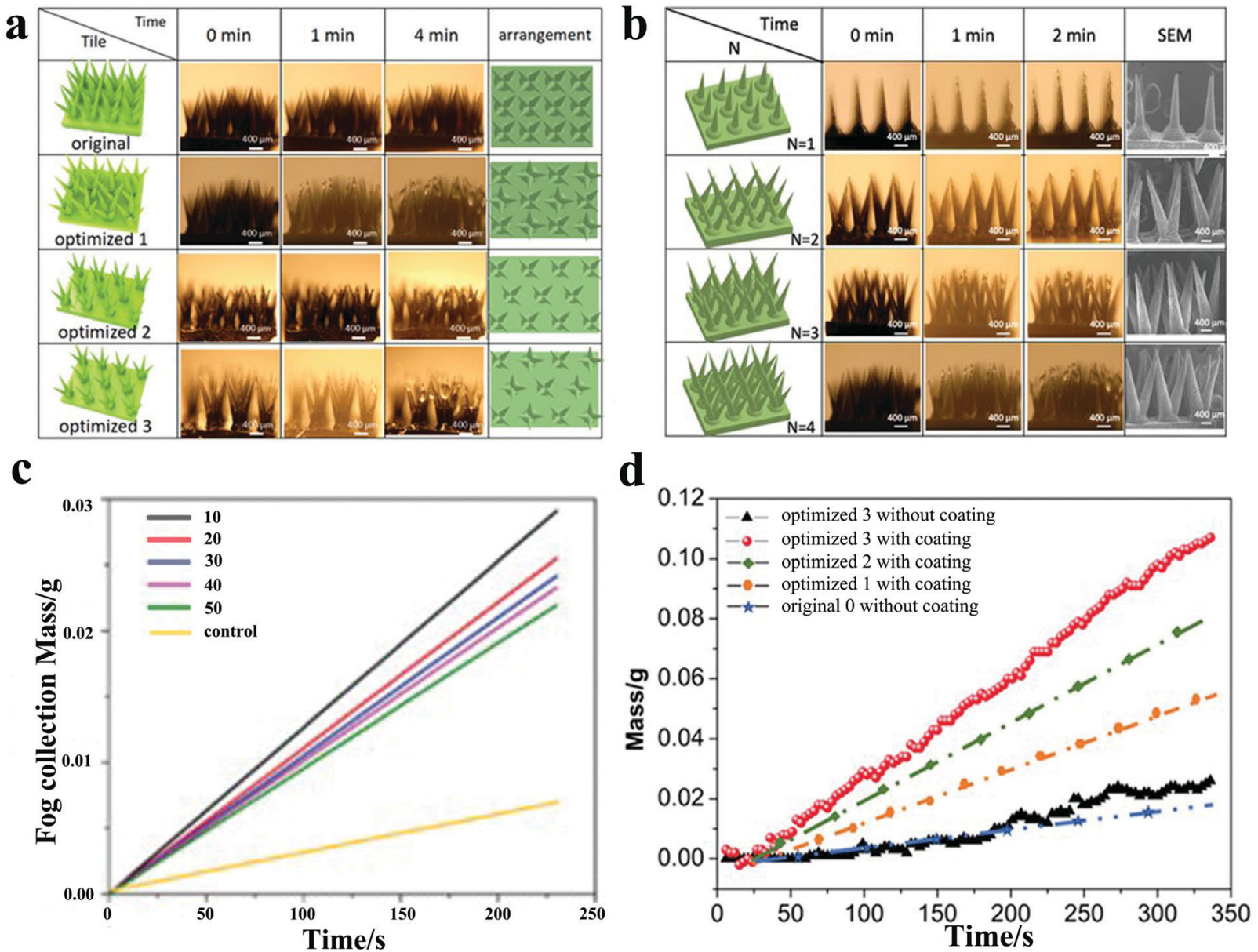

Fig. 5 (a) The water collection process for different arrangements of multi-branched spines with time; optimized 1,2,3 represent the surface with the rotation of multi-branched spines, the movement of multi-branched spines, and the rotation and movement of multi-branched spines, respectively. (b) The water collection process for different number of branched-spine structures with time. (c) Water masses collected by cones with different tip angles with time. (d) The collected water masses for different arrangements of multi-branched spines over time. The surface of optimized 3 with coating has the highest water collection efficiency. Reproduced with permission. ${ }^{79}$ Copyright 2020 , Wiley-VCH.

droplets are captured at the tip and then spontaneously move to the substrate, forming a continuous water flow. On the contrary, the water droplets on bionic fibers just stay on the spindle knots and cannot be fully utilized. In general, the bionic one-dimensional surface is fully exposed to the fog flow, which can maximize the utilization of the structure. After solving the problem of insufficient strength, the bionic onedimensional surface is likely to be applied on a large scale.

\subsection{Bionic two-dimensional surface}

The study of bionic two-dimensional surfaces was inspired by Namib Desert beetles. The design of integrating the hydrophobic area and the hydrophilic area into the same two-dimensional surface improves the water collection efficiency, which is referred to as alternating wettability surface in the following. The water capture, ${ }^{80}$ water supply, ${ }^{81}$ and water removal ${ }^{82}$ are the three processes on alternating wettability surface, whereas surface wettability, surface structure, and hydrophilic-hydrophobic distribution ratio are three factors that affect the water collection efficiency. ${ }^{83}$ According to the distribution of surface wettability, the bionic two-dimensional surface is divided into the following four types: hydrophilic-superhydrophobic surface, superhydrophobic-hydrophilic surface, hydrophilic-superamphiphobic surface, and Janus membrane, which will all be explored below in detail.

3.2.1. Hydrophilic-superhydrophobic surface. The droplets from the wind may be caught when they collide with the superhydrophobic surface. The uniform superhydrophobic surface allows the droplets to maintain high CAs and spherical shape. However, these droplets are unstable and randomly roll on the superhydrophobic surface, which can only be removed by gravity. Gradient and patterning are two important directions in the regulation of the wettability of solid surfaces, which is a common method to realize the directional movement of droplets. ${ }^{84}$ Wetting gradient refers to the continuous change in the wettability of the surface. On the wetting gradient surface, the contact angles of the front and back of the droplet are different, pushing the droplet to move in a more hydrophilic direction. Patterning refers to the construction of a special shape wettability pattern on a solid surface. The wedge-shaped 
wettability pattern is more commonly used to control the direction of droplet movement. On the wedge-shaped patterned surface, the generated Laplace pressure allows the droplet to move from the wedge-shaped tip to the wedge-shaped wide end. ${ }^{85-88}$

Song et al. ${ }^{89}$ combined PDMS and graphene (PDMS/G) to design a smart surface with a sharp shape that could be used for water collection, proving that the geometric pattern and wettability coordination were the key to the directional movement of the droplets. They used spin coating to prepare a superhydrophobic PDMS/G layer on a superhydrophilic glass substrate. Different templates were covered on PDMS/G and the blade was used to cut off the hydrophobic surface under the template to prepare a series of fog collection surfaces with surface energy gradient and shape gradient. In the fog collection test on different wettability surfaces, it was found that the fog droplets collected on the superhydrophilic surface easily formed a water film, which prevented subsequent droplets from being collected. In contrast, on the superhydrophobic surface, individual fog droplets agglomerated into large droplets and were retained on the surface. In the square, tip-shaped, and circular pattern surfaces, the water droplets could be clearly observed to move from the hydrophobic region to the hydrophilic region (Fig. 6a). Compared to the square and round patterns, water droplets moving on the tip-shaped pattern were driven by additional Laplace pressure, which resulted in the tip-shaped patterns collecting more water (Fig. 6b). The conclusion was consistent with what Bai had previously obtained. ${ }^{46}$ It should be added that Bai also discussed the effect of the area distribution ratio of the hydrophilic region to the hydrophobic region on the water collection efficiency, and concluded that the area and shape of the hydrophilic pattern would affect the water collection efficiency.

Apart from engraving hydrophilic patterns on hydrophobic surfaces, the other solution is to grow hydrophilic protrusions directly on the hydrophobic surface. According to Aizenberg's research, the bulges on the back of the desert beetles optimized rapid local droplet growth by gathering the vapor diffusion flux at the apex, proving that the specific topography on a smooth surface could effectively promote water condensation and enhance the vapor diffusion flux..$^{82,90,91}$ Zhong et al. ${ }^{80}$ proved by flow field analysis that these protrusions could change the dynamic pressure distribution above the surface and concentrated the fog flow around the protrusions, which could greatly increase the growth speed of the droplets. The results clearly show that a combination of special surface chemistry and surface morphology can design more effective fog collection
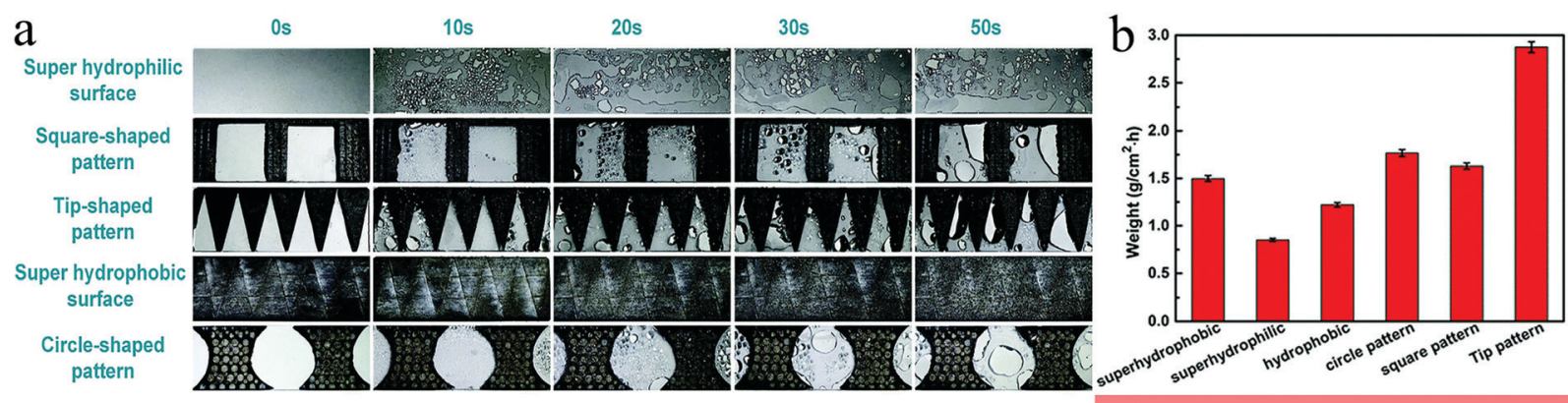

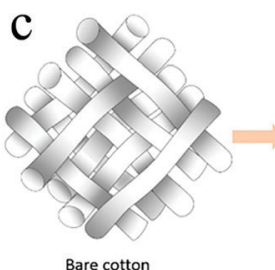

Bare cotton

e

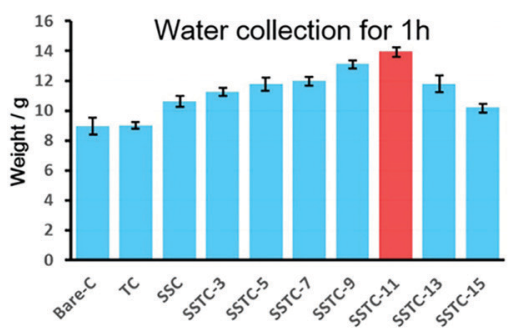

SC

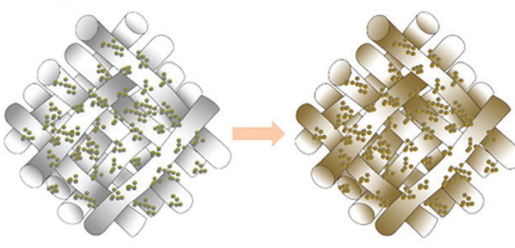

$\mathrm{SiO}_{2} \mathrm{NPS}_{\mathrm{TiO}_{2} \text { domains }}$

$\mathrm{f}$

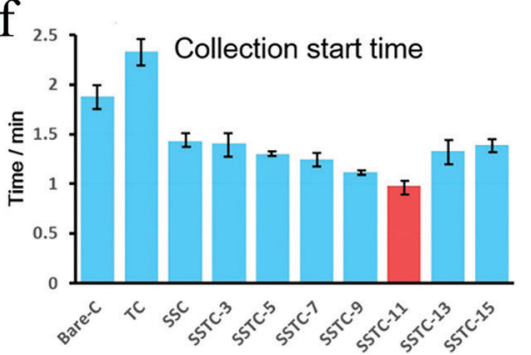

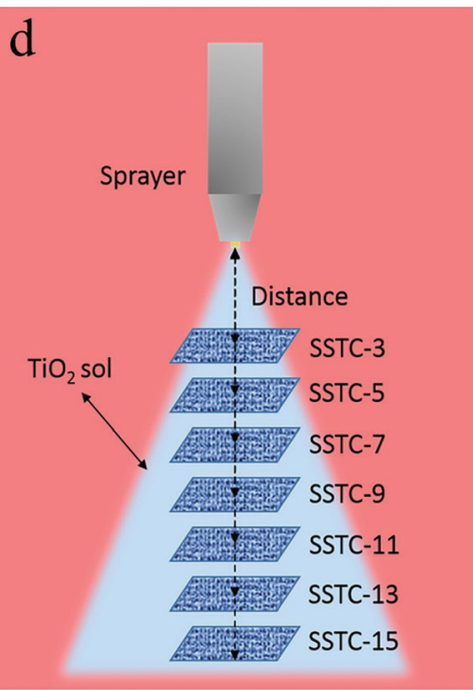

Fig. 6 (a) Fog collection processes on different surfaces (b) fog collection efficiencies of various surfaces. The hydrophilic-superhydrophobic surface with tip patterns exhibited the highest fog collection efficiency. Reproduced with permission. ${ }^{89}$ Copyright 2018, Royal Society of Chemistry. (c) The fabrication process of SC, SSC, and SSTC Fabrics (d) spraying coating $\mathrm{TiO}_{2}$ sol onto SSC by controlling the distance between the nozzle and fabrics (e) the weight of water collected by different fabrics in 1 hour. SSTC-11 showed the highest water collection efficient. (f) The water collection start time of various fabrics. Reproduced with permission. ${ }^{92}$ Copyright 2016, American Chemical Society. 
surfaces. Wang et al. ${ }^{92}$ dip-coated $\mathrm{SiO}_{2} \mathrm{NPs}$ onto bare cotton (C) fabric (SC) by controlling the surface roughness and surface energy, and formed a superhydrophobic SSC fabric after OTES treatment. Finally, a layer of $\mathrm{TiO}_{2}$ nanosol was sprayed. Under the effect of interfacial tension, the nanosol on the superhydrophobic surface produced light-induced superhydrophilic protrusions, thereby forming the SSTC (Fig. 6c). These $\mathrm{TiO}_{2}$ protrusions with wettability gradient and shape gradient could accelerate the coalescence and collection of the water droplets. A series of SSTCs with different spraying distances were manufactured, which had different coating amounts and domain sizes of $\mathrm{TiO}_{2}$ (Fig. 6d). Comparing the weight of water collected by different fabrics after 1 hour and the collection start time, it was found that SSTC-11 (the spraying distance was $11 \mathrm{~cm}$ ) collected the most water (Fig. 6e) and started the collection process faster (Fig. 6f).

In general, by constructing hydrophilic tip patterns and protrusions on superhydrophobic surfaces, the droplets are driven directionally under the influence of surface energy gradient and Laplace pressure, showing better water collection efficiency than surfaces without surface chemical gradient. It should be noted that the area ratio of the hydrophilic region to the hydrophobic region is also the key to the efficiency of water collection.

3.2.2. Superhydrophobic-hydrophilic surface. In addition to constructing hydrophilic-superhydrophobic surfaces, constructing superhydrophobic-hydrophilic surfaces is also feasible for fog harvesting. However, as far as we know, this method is rarely mentioned. From the perspective of nucleation growth, the hydrophilic surface is conducive for droplet nucleation growth. Theoretically, the nucleation density of droplets on a hydrophilic surface may be an order of magnitude greater than that on a hydrophobic surface, and this is also confirmed from the data collected. ${ }^{82,93,94}$ The hydrophilic surface also has a much larger coalescence rate than the hydrophobic surface, which is reflected in the rapid growth rate of the droplets. It can be explained by three reasons: ${ }^{95}$ First, due to the low contact angle, compared to the hydrophobic surface, the droplets exhibit a larger contact area in hydrophilicity, which reduces the effective distance between the droplets and promotes the merging of droplets. Second, higher nucleation density causes the droplets to coalesce faster. Third, on some hydrophobic surfaces using lubricants, small water droplets may form a coating layer at the water-air interface, which forms non-coalesced droplets. However, as the coalescence process continues, if not removed in time, the droplets will easily form a water film on the hydrophilic surface. Due to the low heat transfer of the water film, it is difficult for the droplets in the mist to continue to condense. It even rebounds from the surface of the water film..$^{77}$ In contrast, the hydrophobic surface has a higher contact angle and a lower initial nucleation rate but the sustained steady-state coagulation rate is higher. ${ }^{96}$ From the point of view of droplet removal, it is much easier for a droplet to leave hydrophobic surfaces than hydrophilic surfaces. Water droplets behave as continual dropwise condensation on superhydrophobic surfaces. The surface energy released when the droplets coalesce is another force that drives the movement of the droplets except gravity. ${ }^{97-99}$ In particular, during steam condensation, the force can even cause the droplets to leave the surface in a jumping form. ${ }^{100,101}$ In addition, the adhesion of the hydrophobic surface is generally low; the droplets in the form of spheres are less resistant to rolling on it, which is beneficial for refreshing the water collection surface. ${ }^{102}$ In the directional movement of droplets, it is important to reduce the resistance to movement. On the hydrophilic surface, water droplets are in the Wenzel state, which strengthens the pinning effect and is not conducive for the movement of the droplets. ${ }^{103}$ The pinning of the rear end of the droplet is the source of resistance of the droplet movement and is related to the pinning phenomenon of the contact line. It will cause the droplets to slow down or even make the contact line completely pinned to the surface, which can only be spread forward and cannot be completely moved.

Yin et al. ${ }^{104}$ solved the problem that the droplets on the hydrophilic surface could not be refreshed in time by constructing a superhydrophobic copper mesh on the hydrophilic surface. The hydrophobic copper mesh surface with polytetrafluoroethylene (PTFE) nanoparticles was deposited by femtosecond laser direct writing technology and then the treated copper mesh was closely combined with a hydrophilic copper sheet (Fig. 7a). Compared with other samples (original copper mesh (hydrophobic), original copper sheet (hydrophilic), treated copper mesh (superhydrophobic), and original copper mesh sheet (hydrophobic-hydrophilic)), the treated copper mesh-sheet exhibited enhanced water collection rate (WCR) (Fig. 7b). At the same time, changing the mesh number of the copper mesh and the inclination angle of the substrate, it was found that the sample using the 40-mesh copper mesh at an inclination angle of $90^{\circ}$ showed the highest WCR and the WCR decreased with the increase in the mesh number (Fig. 7d). Driven by the wettability gradient, the droplets deposited on the superhydrophobic copper mesh moved to the hydrophilic copper plate and grew up. Due to the high contact angle on the copper mesh, the droplets with a diameter exceeding the copper mesh would easily fall under the action of gravity (Fig. 7c).

The superhydrophobic-hydrophilic hybrid surface effectively balances the adsorption and removal processes of the droplets, opening up a new way for constructing hybrid water-collection surfaces on hydrophilic substrates. However, compared with the hydrophilic-superhydrophobic surface, the WCR displayed by this water collection method is not as high as that of the former. One point that can be improved is to get an optimal wettability combination by changing the difference in the contact angle between the hydrophobic copper mesh and the hydrophilic surfaces. In addition, the timely removal of water on hydrophilic surfaces will greatly improve the collection efficiency of superhydrophobic-hydrophilic surfaces.

3.2.3. Hydrophilic-superamphiphobic surface. Superamphiphobic surface refers to a surface with a contact angle of more than $150^{\circ}$ with water and oil. ${ }^{105}$ In natural conditions, the fog collection surface may be contaminated with dust and grease, which may cause the local wettability of the system to be changed and the water collection performance will be adversely affected. 

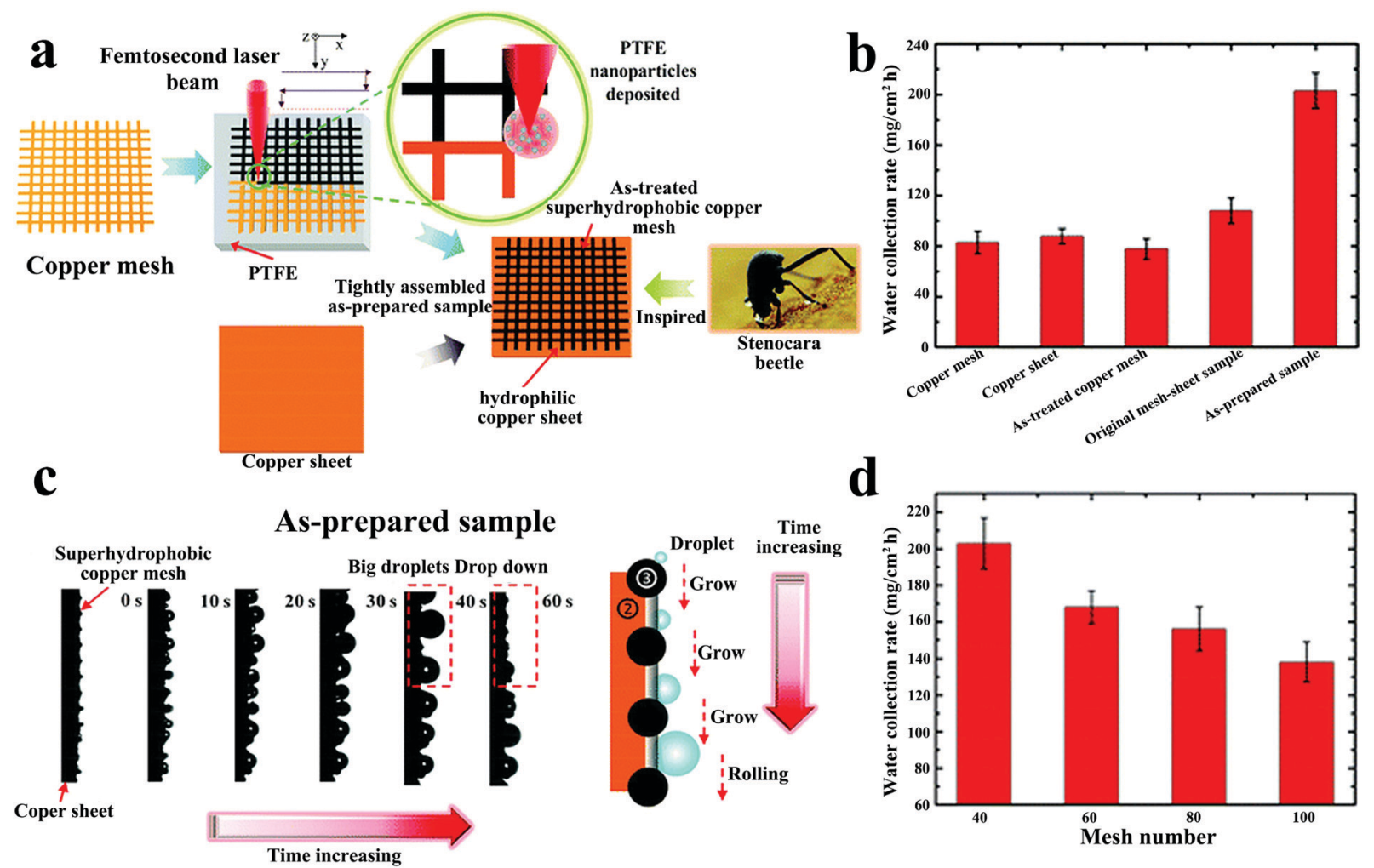

Fig. 7 (a) The preparation for the superhydrophobic-hydrophilic hybrid surface. (b) The water collection rates of various samples. (c) The movement process of water droplets on superhydrophobic-hydrophilic hybrid surface. (d) The relationship between copper mesh number and water collection rate. Reproduced with permission. ${ }^{104}$ Copyright 2017, Royal Society of Chemistry.

The application of superamphiphobic surface is expected to solve this problem. ${ }^{106}$ Wang et al. ${ }^{37}$ prepared a series of internally developed chain-shaped nano-SiO ${ }_{2}$ superamphiphobic coatings mixed with various hydrophilic particles for fog harvesting. The five different particles were $\mathrm{SiO}_{2}$ nanoparticles, hydrophobic SiC particles, SiC particles wrapped with PAA, SiC particles wrapped with PVA, and SiC particles wrapped with KH550. The corresponding water collection surfaces were called SOS, SCS, SAS, SVS, and SHS. These particles coated with hydrophilic polymer were composed of hydrophilic and hydrophobic regions; thus, their water contact angle was higher than that of the polymer. After spraying these particles on the hybrid surface, the water/oil contact angle and rolling angle of different surfaces were measured respectively, as shown in Fig. 8a and b. The water and oil contact angles of all hybrid surfaces were greater than $150^{\circ}$, and the rolling angles were less than $5^{\circ}$, which indicates that the hybrid surface showed excellent superhydrophobicity and superoleophobicity. When conducting the condensation test, it was found that the coated particles had better nucleation ability than the uncoated particles. This was due to the increased wettability of the hydrophobic SiC particles when they were coated with the hydrophilic polymer, which gave the surface enhanced droplet nucleation ability. In addition, in the water collection test, the SAS surface showed the highest water collection efficiency (Fig. 8c). Fig. 8d shows the enhanced droplet nucleation and droplet removal mechanism on the hybrid surface. The surface energy released by the droplets during the coalescence of the vertical surface overcomes the dissipation of viscosity $\left(E_{\mathrm{vis}}\right)$ and work of adhesion $\left(E_{\mathrm{w}}\right)$. Under the action of gravity, the droplets easily left the surface in the form of jumping, which greatly enhanced the droplet removal ability. The combination of high nucleation and droplet removal efficiency resulted in excellent water collection rate on the SAS surface.

When discussing the influencing factors of WCR, the rolling angle (RA) should not be ignored. This work proved that when RA was less than a special value, WCR and RA were positively correlated. In addition, compared with the hydrophilic-superhydrophobic surface, the hydrophilic-superamphiphobic surface can effectively resist oil pollution. It provides an important reference for the production of oil-proof, self-cleaning water collection surfaces.

3.2.4. Janus membrane. Both sides of the Janus membrane have different properties. ${ }^{107-111}$ In interface chemistry, the most commonly used method is to make a Janus membrane with different wettability, such as a hydrophobic-hydrophilic Janus membrane. Compared with traditional membranes, Janus membranes significantly reduce the energy loss of water droplets during transportation. Due to the surface tension and hydrophobic effect of water, the water droplets always remain spherical on the hydrophobic side. Under the capillary force, water droplets enter the other side of the membrane through small holes. The water droplets are quickly pulled from the hydrophobic area to the hydrophilic area under the effect of the 

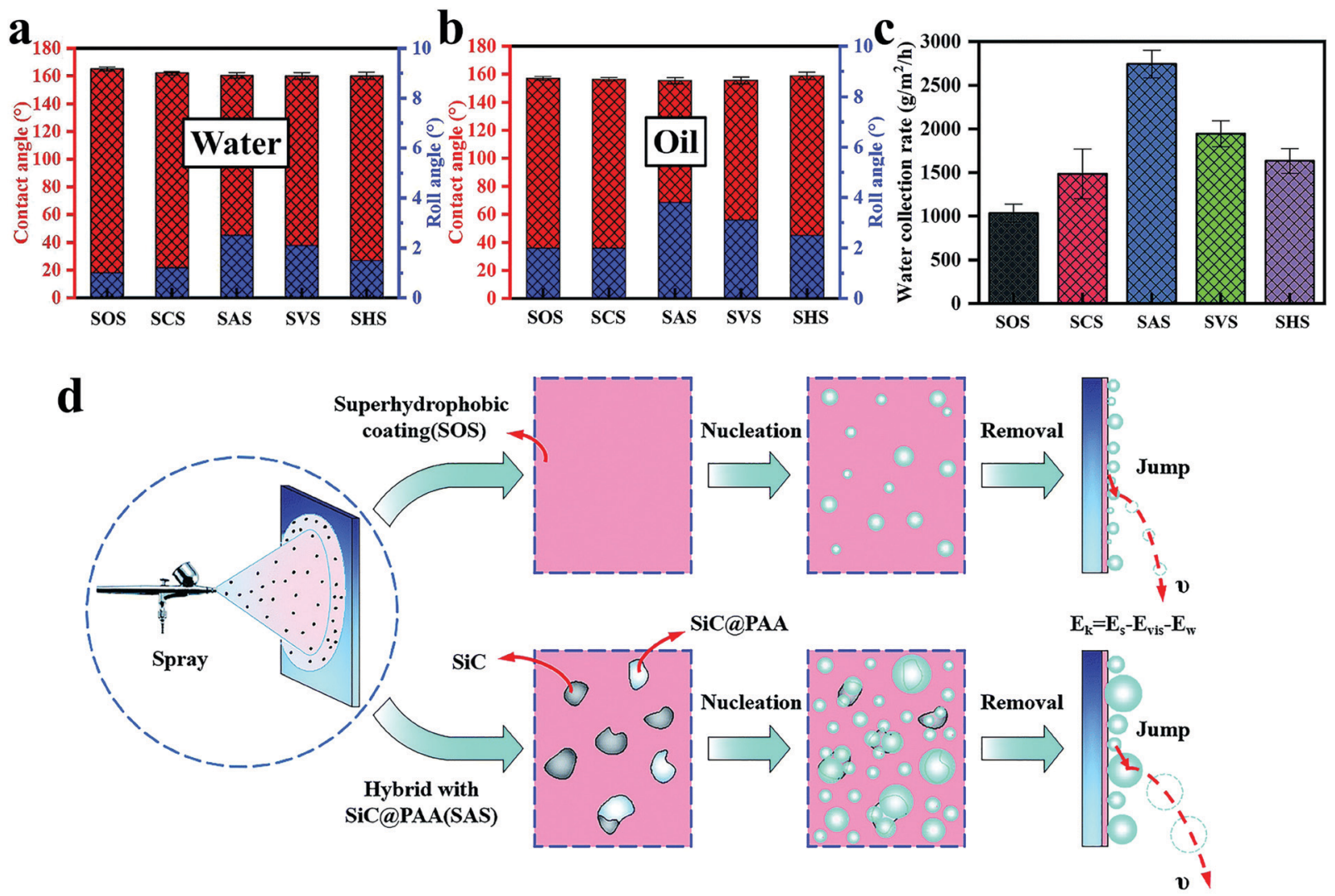

Fig. 8 (a) The contact angle and rolling angle of water droplets on various surfaces. (b) The contact angle and rolling angle of oil droplets on various surfaces. (c) The water collection rate on various surfaces. (d) The principle of enhanced nucleation and droplet removal. The hybrid surface composed of superamphiphobic surface and polymer-encapsulated particles produces a high nucleation rate and excellent droplet removal efficiency. Reproduced with permission. ${ }^{37}$ Copyright 2019, Royal Society of Chemistry.

wetting force gradient, thereby achieving spontaneous directional transportation. ${ }^{112}$ The process is irreversible; thus, the Janus membrane is also called "liquid diode", ${ }^{113}$ which has important applications in the fields of water collection, ${ }^{104}$ oil-water separation, ${ }^{114-117}$ membrane distillation, ${ }^{118,119}$ and sensors. ${ }^{120,121}$ The preparation of water collection equipment should meet some requirements: relatively hydrophobic surfaces, rapid transport of water droplets, rapid surface refresh, and rapid collection. ${ }^{122}$ Due to its unidirectional transmission mechanism, the Janus system helps the water droplets to quickly transport and release dry surfaces, which greatly improves the efficiency of fog collection.

Cao et al. ${ }^{122}$ made a hydrophobic-hydrophilic cooperative Janus system by combining hydrophobic copper mesh-modified with dodecyl mercaptan and hydrophilic cotton. The system proved that the Janus system could effectively increase the water transportation speed and reduce the water re-evaporation rate. In addition, making the system into a $3 \mathrm{D}$ cylinder could prevent the collector from being disturbed by the direction of fog flow and successfully complete the water collection process at any location. Zhou et al. ${ }^{123}$ proposed a micro/nano-conical spine and Janus membrane-integrated system (MNCS + JM) by combining the cactus spine and Janus system. In this system, MNCS was covered with rough micro-nano structures, which imitated the cactus to collect the mist droplets, while the Janus membrane transported the aggregated droplets to the other side. The droplet collection process could be divided into the following steps: tip capture, transmission and coalescence on the stem, and penetration on the JM (Fig. 9a). The fog droplets collided with the spine and were captured by the tip. Due to the gradient of curvature, the droplets at the tip of the MNCS were driven by the Laplace pressure to move to the end and merge (Fig. 9b). When they touched the hydrophobic surface of the JM, the hydrophobic force $\left(F_{\mathrm{HF}}\right)$ would prevent the droplets from permeating but the more powerful hydrostatic pressure $\left(F_{\mathrm{HP}}\right)$ generated by the wettability gradient drove them to move quickly from the hydrophobic side to the hydrophilic side. In addition, the water droplets released the surface energy when they reached the JM, which was also conducive for the efficient penetration of the droplets (Fig. 9c). In the water collection test, by adjusting the contact angle range of the hydrophilic surface of JM and the effective length of MNCS, it was found that the increase in the hydrophilicity of the hydrophilic side of JM and the decrease in the length of MNCS could further optimize the water collection efficiency. Comparing the relationship between the droplet circulation rate and the water collection rate, the water collection rate was roughly proportional to the droplet circulation rate. The faster the circulation 


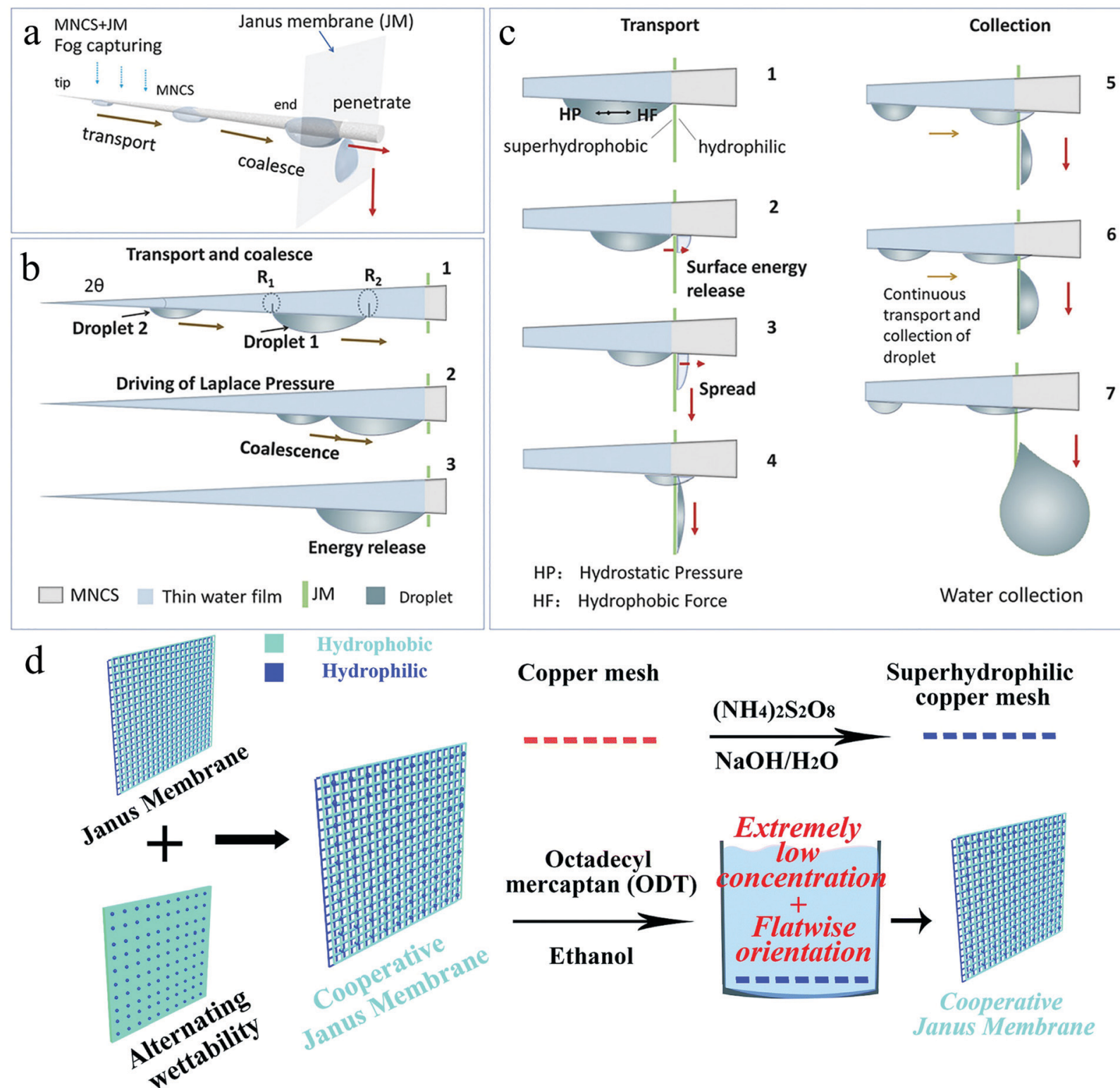

Fig. 9 (a) The fog collection process of MNCS + JM, which includes capture, transport, coalescence, and penetration. (b) The droplets are transported and coalesced under the influence of Laplace pressure, and release surface energy when combined. (c) When the droplet touches the surface of the JM, HP prevents its penetration and the stronger HF promotes its penetration. The released surface energy also facilitates the penetration of the droplets. Reproduced with permission. ${ }^{123}$ Copyright 2018, Wiley-VCH. (d) Illustration of Cooperative Janus Membrane preparation process. Reproduced with permission. ${ }^{124}$ Copyright 2019, Royal Society of Chemistry.

speed, the higher the water collection rate. Importantly, the water collection rate could also be increased by increasing the number of MNCS spines.

Zhong et al. ${ }^{124}$ integrated a nano-scale hydrophobic-hydrophilic alternately wettable surface with the Janus system through simple liquid line modification to obtain enhanced water collection capacity. Through the ammonia etching method, the copper mesh was modified into a superhydrophilic copper mesh covered with $\mathrm{Cu}(\mathrm{OH})_{2}$ nanoribbons, which was then immersed in the 1-octadecanethiol/ethanol mixed solution (Fig. 9d). By controlling the modification time, the sample placement position, and the mixed solution concentration, four types of Janus copper mesh with different surface wettabilities were prepared: $\mathrm{SHH} / \mathrm{SHH},(\mathrm{HH}+\mathrm{HB}) / \mathrm{HH}, \mathrm{SHB} / \mathrm{SHB}$, and $\mathrm{HH} / \mathrm{HH}$, where $\mathrm{SHH}, \mathrm{HH}, \mathrm{HB}$, and SHB represent superhydrophilic, hydrophilic, hydrophobic, and superhydrophobic, respectively. In the mist collection test, it was found that the $(\mathrm{HH}+\mathrm{HB}) / \mathrm{HH}$ mode JM had the highest water collection rate. The advantages of HH-HB alternately wettable surfaces have been discussed previously. The water droplets deposited and coalesced on the HB point, and then moved to the $\mathrm{HH}$ point and penetrated to the other side under the action of the asymmetric wetting force. The rapid water removal method was independent of gravity and more effective. In summary, the integration of different mechanisms on a copper 
mesh showed enhanced capture, transmission, and removal of fog. Compared with the amount of water collected by the corresponding superhydrophilic membrane, the enhanced JM's water collection rate increased to $800 \%$. Compared with the alternating wettability surfaces discussed earlier, the water droplets received an additional driving force on the surface of the Janus membrane. Hydrostatic pressure and capillary force cooperated with the asymmetric wetting force to drive water droplets to penetrate from one side to the other. The method accelerated the transfer process of the water droplets and helped the fog collection surface to remain dry, thereby realizing the continuity of the fog collection process. During the preparation process, it should be noted that the difference between the thickness of the hydrophobic side film and the wettability at both ends is the key to the droplet penetration rate.

In a word, the bionic two-dimensional surface is a mature microstructure design for fog collection. To improve the WCR, it is essential to modify the two-dimensional surfaces into alternating wettability surfaces. In addition, patterning and structuring help the water droplets to obtain additional Laplacian driving force. First, the WCR of the gradient wetting surface with special patterns and structures is significantly higher than that of ordinary hybrid surfaces. Secondly, the area ratio of the hydrophobic region to the hydrophilic region is an important factor influencing WCR and the superhydrophobichydrophilic mode should be considered preferentially. Finally, the timely removal of water adhered to the surface is a key step for improving the WCR. The unidirectional penetration of water droplets in the Janus system is conducive for the continuous refreshing of the fog collection surfaces; thus, great attention should be paid when manufacturing the bionic twodimensional fog collection surface.

\subsection{Three-dimensional composite structure}

Fog droplets must undergo multiple processes during water collection and a single mechanism often cannot suit the needs at the same time. For example, the deposition speed of water droplets on hydrophilic surfaces is very fast but due to the low contact angle, it is easy to form a water film and hinder the next round of water collection. ${ }^{89}$ Water droplets are spherical on the hydrophobic surface, which are easy to coalesce with the surrounding droplets to form large droplets but the nucleation speed is relatively slow. Three-dimensional composite structures greatly improve the fog collection efficiency by integrating the advantages of these mechanisms.

Zhang et al. ${ }^{125}$ integrated the water collection mechanism of spider silk and cactus to prepare a three-dimensional fiber network $\left(\mathrm{N}_{3} \mathrm{D}\right)$ decorated with nano-cones. The fiber network was woven from hydrophobic lightweight nylon and then hydrophilized $\mathrm{ZnO}$ nano-cones were grown on the fiber network. During the water collection process, the water droplets were captured on the hydrophilic ZnO nano-cones, which were quickly moved from the tip to the base and fibers under the drive of the Laplace pressure difference. The continuous process made the nano-cones covered with a film of water (Fig. 10a). When the thickness of the water film reached a certain value, due to Rayleigh instability, it would automatically decompose into droplets and most of the droplets formed a rapid continuous water flow on the hydrophilic nano-cone (Fig. 10b). The water flow gathered on the fiber network; as the weight increased, water droplets automatically fell off the $\mathrm{N}_{3} \mathrm{D}$ surface (Fig. 10c). It is a novel water collection mechanism that speeds up the capture and transportation of fog droplets. Compared with the conventional intermittent water collection method, the method achieved an automatic continuous water collection process. Under the same conditions and time, the water collection of $\mathrm{N}_{3} \mathrm{D}$ was 2-3 times that of the original net. Within two hours, the mass of water collected using $\mathrm{N}_{3} \mathrm{D}$ reached more than 240 times the mass of the mesh sample itself and the maximum collection efficiency reached $865 \mathrm{~kg} \mathrm{~m}^{-2}$ per day. The result shows that after integrating multiple mechanisms, the application of $\mathrm{N}_{3} \mathrm{D}$ in the water collection equipment can effectively improve the efficiency.

Hu et al. ${ }^{126}$ combined the asymmetric structure of the cactus spines and the alternate wetting structure of the desert beetle to design a hybrid membrane with an asymmetric microscopic topology and anisotropic wetting. The hybrid membrane was composed of a hydrophilic nanoneedle layer and a hydrophobic nanofiber layer (Fig. 10d). Polyvinylidene fluoride-co-hexafluoropropylene (PVDF-HFP) nanofibers (PNFs) were covered on the copper mesh by electrospinning to form a hydrophobic nanofiber layer. Then, through electrochemical anodizing treatment, a layer of hydrophilic $\mathrm{Cu}(\mathrm{OH})_{2}$ nanoneedles (CNN) was obtained. By changing the electrospinning time and electrochemical anodizing time, the length of CNN and the water permeability of the hybrid membrane could be controlled. There were two modes to defog the hybrid membrane (Fig. 10e). One was that the droplet touched the interweaving area of CNN/PNF. Due to the hydrophilic and asymmetric shape of the copper needle, the droplets were transported to the bottom of the CNN under the influence of Laplace pressure and directly absorbed by the copper mesh. The other mode was that the droplets were captured by the hydrophobic PNF and slowly coalesced to grow. When the edge of the water droplet touched the CNN, it was quickly absorbed by the CNN grid under the influence of the hydrophilic capillary force and the Laplace pressure difference. These two processes were irreversible because the Laplace pressure and gradient wetting force prevented the collected water from passing through the PNF grid micropores. In the fog collection test, the optimal electrospinning time and anodizing time were 5 minutes and 10 minutes, respectively. The corresponding water collection efficiency was $0.116 \mathrm{~g} \mathrm{~min}^{-1} \mathrm{~cm}^{-1}$, which has been an excellent efficiency value in recent years.

According to Krishnan's research, water droplets were simultaneously subjected to the force of capillary and wetting gradient in the plant microchannel, allowing rapid passage and transmission. ${ }^{127}$ Wang et $a .^{73}$ combined the water collection mechanism of cactus and Sarracenia to achieve drop-by-drop capture and ultra-fast water transmission. Sarracenia has a layered micron/nano scale structure, which can be applied for the directional transport of water. Because of its unique layered microchannel organization of trichomes, the water transfer 

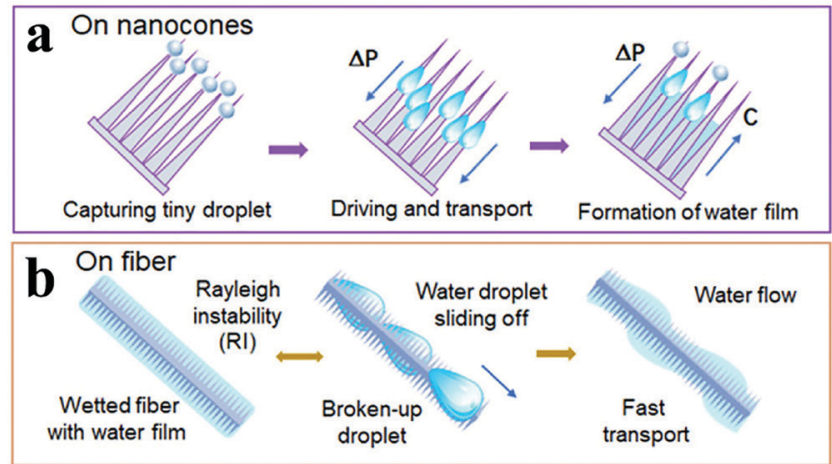

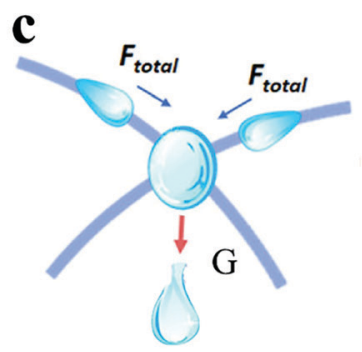

High efficiency Water sliding off

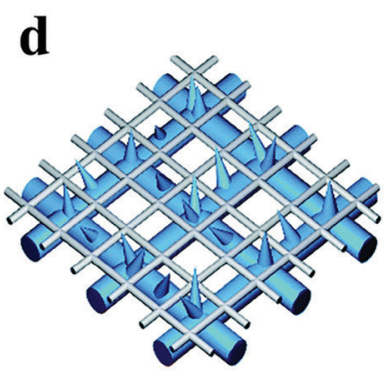

CNN/PNF hybrid membrane
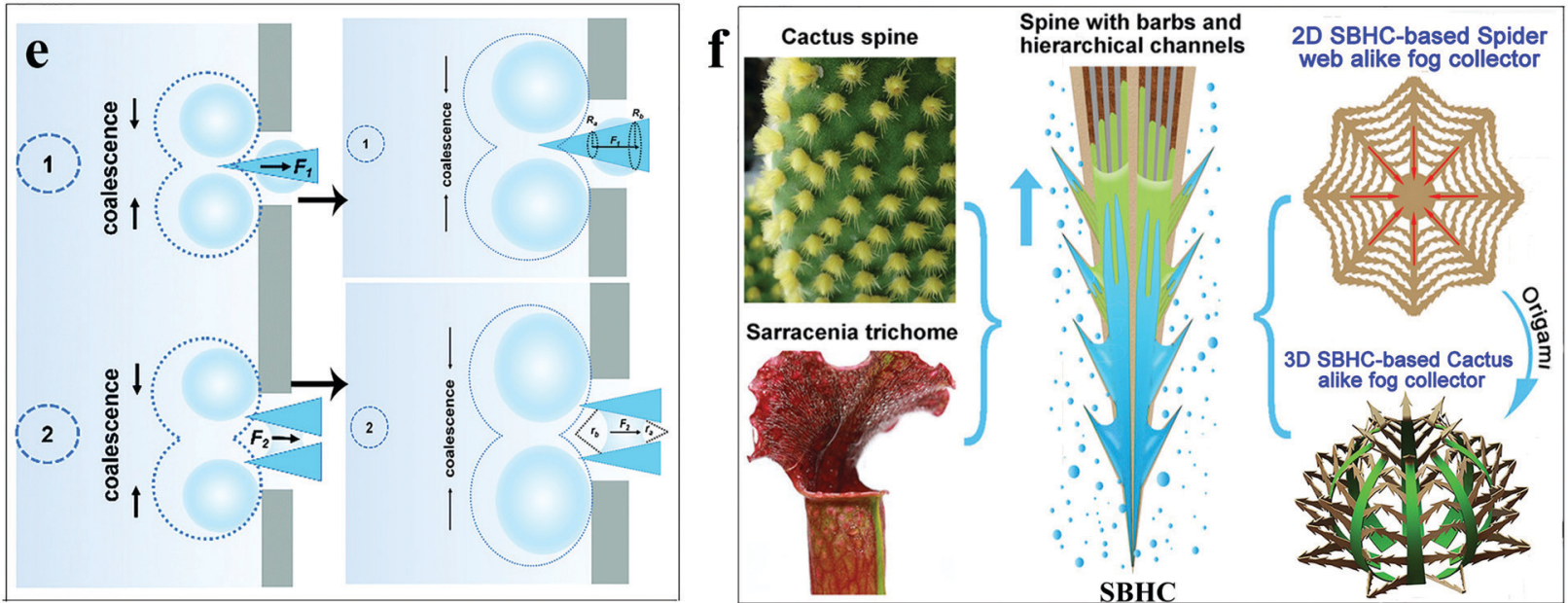

Fig. 10 (a) The process of forming a water film on nano-cone clusters. (b) Due to Rayleigh instability, the water film automatically decomposed into droplets and most of the droplets formed a rapid continuous water flow on the hydrophilic nano-cone. (c) Water flow gathered at the intersection of fibres, sliding off under the action of gravity. Reproduced with permission. ${ }^{125}$ Copyright 2019. American Chemical Society. (d) Illustration of the CNN/PNF hybrid membrane. (e) Two modes of hybrid membrane defogging. Reproduced with permission. ${ }^{126}$ Copyright 2019 , Royal Society of Chemistry. (f) Design illustration of the spider web-like fog collector based on 2D SBHC and the alike fog collector based on the 3D SBHC. Reproduced with permission. ${ }^{73}$ Copyright 2020, American Chemical Society.

speed is three orders of magnitude faster than that on the cactus spine and spider silk. ${ }^{128} \mathrm{~A}$ series of spine (SBHC) with barbs and grading channels on the surface of the copper sheet were created by laser direct construction technology to simulate the thorns of cactus and Sarracenia (Fig. 10f). Compared with the spine with barbs and grooves (SBG), the spine with hierarchical channels (SHC), and the spine with only barbs (SB), SBHC exhibited the best defogging performance. There were eight barbs distributed on the spine of each SBHC unit, and layered microchannels with high and low ribs were distributed on the surface of the spine and aligned with the barbs. There were two possible ways to transport water droplets on the surface of SBHC. One was the transmission along the dry layered channel and the other was the slip along the wet water film. The rough surface of the graded microchannel greatly enhanced the wettability of the microchannel, which resulted in a strong capillary force on the concave meniscus between the spine and the barb. The capillary force and the Laplace pressure together drove the rapid transport of water droplets. 96 SBHC units were integrated with a spider web with a diameter of $5 \mathrm{~cm}$ to form a 2D SBHC mesh water collector. Due to the ultra-fast water transfer speed, the water droplets deposited on the SBHC unit are quickly transported to the center of the web and slide down under the action of gravity. The WCR of 2D spider web-like fog collector reached $0.08 \mathrm{~mL} \mathrm{~min}^{-1}$. Correspondingly, making $96 \mathrm{SBHC}$ units into a 3D cactus-like fog collector could also achieve spontaneous rapid water collection; the WCR was $0.09 \mathrm{~mL} \mathrm{~min}^{-1}$. It indicates that the SBHC fog collector that integrated the micro/nano channel and the asymmetric structure of the cactus spine successfully obtained fast water transmission speed and excellent WCR.

The above data shows that after combining the water collection mechanism of spider silk, cactus, desert beetle, and bottle grass, the corresponding WCR was greatly improved. However, most of the existing water collection surfaces only combined two mechanisms. To obtain higher WCR, water collection equipment with more mechanisms should be explored.

In summary, we discussed the water collection mechanism and design method of bionic one-dimensional surface, bionic two-dimensional surface, and three-dimensional composite structure. In the one-dimensional structure, the water collection mechanism of bionic fibers and asymmetric conical structures is relatively simple, and the corresponding WCR is low. The hydrophilic-hydrophobic mode on bionic two-dimensional 
surfaces provides a strong driving force for water droplets, which is conducive for the directional movement of water droplets. At the same time, the WCR is also improved due to the larger fog collection area. The three-dimensional composite structure integrates a variety of water collection mechanisms, which results in faster water transportation speeds and higher WCR. A composite structure is the most potential way to improve the fog collection efficiency in the future. The working environment of the water collection equipment is extremely harsh, requiring high reliability and recyclability. Next, we will focus on how to improve the sustainability of the water harvesting surface as it helps to design and prepare water collection equipment with excellent natural tolerance.

\section{Sustainability of the water collection surface}

The water collection equipment may be subject to various challenges from harsh natural environments. It may be contaminated by dust in the wind, abraded by sands, corroded by acid rain, polluted by the proliferation of bacteria, and age due to long-term ultraviolet radiation. In order to resist these threats and maintain high water collection efficiency, the water collection surface must be sustainable. Here, we discussed how to improve the mechanical stability, chemical stability, and antibacterial properties of the water collection surface.

\subsection{Mechanical stability}

The hydrophobic area is an indispensable part of the water collection surface. It helps the droplets to maintain a spherical shape on the surface, which is beneficial for the droplet growth and removal from the surface. However, most of the hydrophobic surfaces with microscopic surface morphology show poor mechanical stability and their fragile surface texture can be easily removed even by rubbing with fingers. ${ }^{129}$ It is very detrimental to the water collection process because the destruction of the hydrophobic surface micro-morphology means the lack of hydrophobicity, which may cause the surface wettability to change from hydrophobic to hydrophilic and the water collection efficiency will also be greatly reduced. ${ }^{130}$ The development of mechanically durable hydrophobic surfaces is very important for extending the life of the water collection equipment. At present, there are a few superhydrophobic surfaces that exhibit mechanical durability. After experiencing sand impact, ${ }^{47,131-133}$ tape peeling test, ${ }^{134}$ wiping with a soft cloth, ${ }^{135-137}$ and abrasive paper, ${ }^{138-140}$ the water droplets still maintained a high contact angle. Unfortunately, these stabilities cannot last too long and after a large cycle value, the hydrophobicity will still decrease.

Wang et $a .^{141}$ constructed a superhydrophobic surface with an armored structure through a hierarchical scale. The superhydrophobicity was provided by the nanostructure and the microstructure provided the robustness. The microstructure was a micro-inverted pyramid-shaped cavity fabricated on a silicon substrate by photolithography and encapsulated fluorinated nanoclusters that provided superhydrophobic silica. When the armored structure was exposed to external forces, the interconnected microstructures blocked objects larger than the frame size, thereby protecting the nanostructures (Fig. 11a). The mechanical stability of the microstructure was determined by the geometry. Generally, increasing the angle $\alpha$ between the side wall of the microstructure and the substrate is a method for improving the structural stability but it also increases the liquid-solid contact fraction $\left(f^{\text {micro }}\right)$, which affects the surface hydrophobic properties. When $\alpha$ was $120^{\circ}$, the mechanical stability and hydrophobic performance reached the best balance point. On repeatedly scraping the surface with a steel knife, the nanostructure maintained a high degree of integrity. It was worth noting that abrasion removed the fluorinated silane layer on top of the microstructure, which changed the local wettability of the surface from hydrophobic to hydrophilic (Fig. 11b). Overall, the gas-liquid-solid composite interface was very stable, which was due to the excellent anti-friction performance and mechanical stability provided by the microstructure (Fig. 11c). In order to better evaluate the effect of mechanical wear on superhydrophobic surfaces, the static contact angle $\theta^{*}$ and rolling angle $\theta_{\text {roll }}$ of the surface before and after wear were measured successively under different opening widths $l$ and $f^{\text {micro }}$ (Fig. 11d and e). The data showed that the static contact angle of the surface decreases slightly and the rolling angle increases slightly after wear but, overall, the surface still maintained a high degree of hydrophobicity. Compared with the Cassie-Baxter model, the results were highly similar, which illustrated that the hydrophobic performance was not affected by the size of the inverted pyramid structure and the armored structure could provide excellent protection for the nanostructure.

In the literature regarding fog collection, there are a few tests for mechanical durability. Most of the fog collection surfaces prepared by chemical etching, spraying, 3D printing, etc., are relatively fragile and cannot withstand mild friction experiments. The highly wear-resistant hydrophobic armored structure is expected to be applied in water collection systems to protect the equipment from outdoor sand erosion, extend the service life, and improve the equipment stability. In addition, using wear-resistant substrates and improving the spraying process are also considered as methods for improving the mechanical stability.

\subsection{Chemical stability}

The working environment of water-collecting materials is full of uncertainty. Environmental pollution may make rainwater acidic and corrode the surface of the catchment, causing it to lose its original chemical properties. Long-term exposure to high temperature and strong ultraviolet rays is also an important factor that threatens the chemical stability of the water collection surface. Therefore, a reliable outdoor water collection surface should have good chemical stability so as to be able to resist a certain degree of acid and alkali corrosion, high temperature and low temperature, and strong ultraviolet radiation. ${ }^{48}$

Song et al. ${ }^{142}$ developed a mixed wetted surface combining PDMS and graphene (PDMS/G) on the copper mesh, where the 

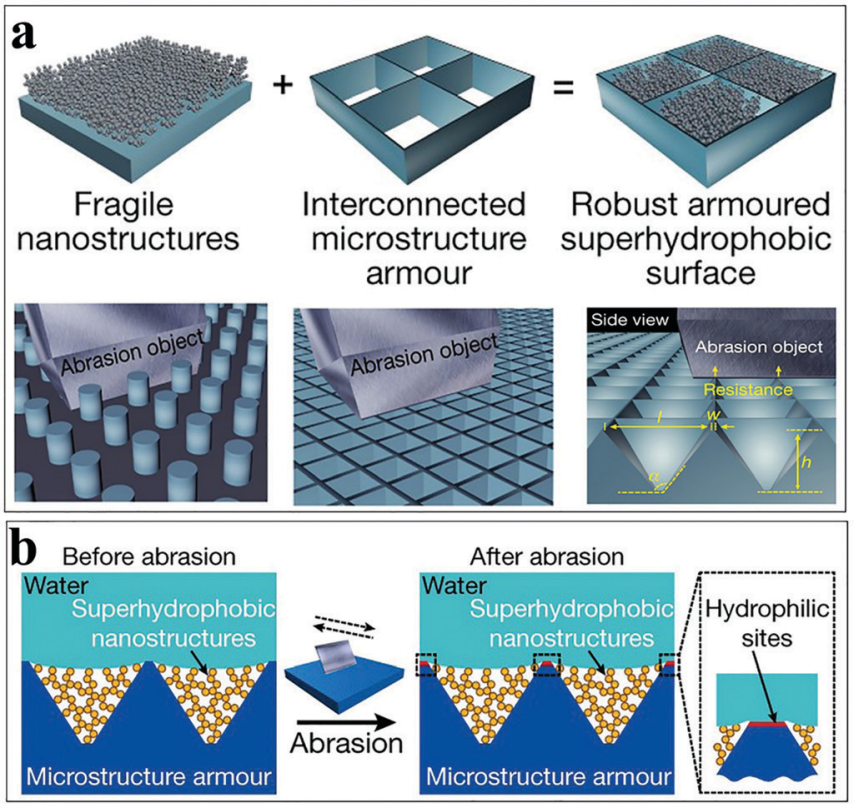

C

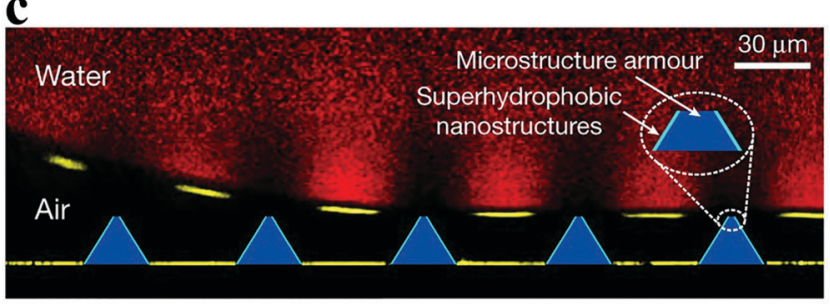

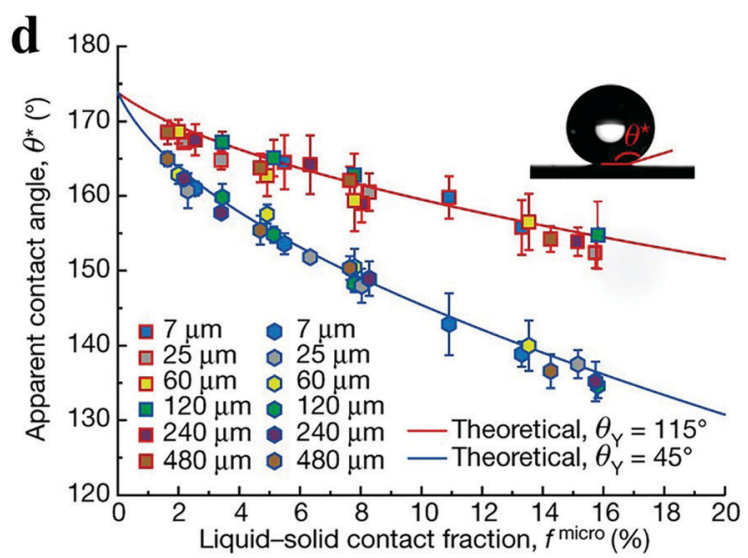

e

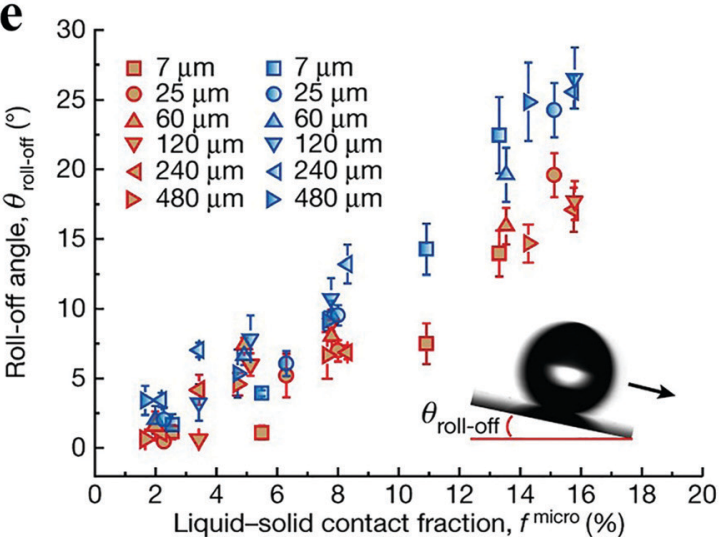

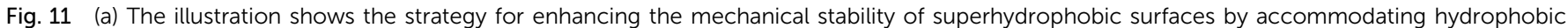

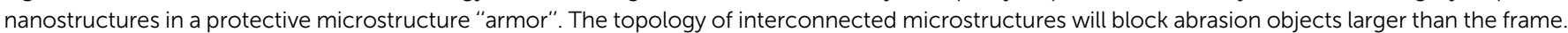

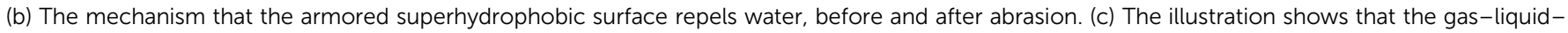

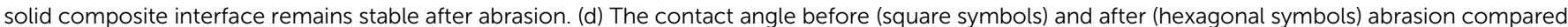
with the theoretical model. (e) The rolling angle before (red) and after (blue) abrasion. Reproduced with permission. ${ }^{141}$ Copyright 2020 , Nature.

mass fraction of graphene powder was 3.3\%. After the PDMS/G film was deposited on the copper mesh surface by laser etching, ultrasonic vibration was performed to obtain a mixed wetted film with gradient wettability. In the corrosion resistance test, the mixed wetted copper mesh was immersed in $1 \mathrm{M} \mathrm{HCl}, 1 \mathrm{M}$ $\mathrm{NaOH}$, and $1 \mathrm{M} \mathrm{NaCl}$ solution for 2 hours (Fig. 12a), and then the wettability (Fig. 12b) and WCR of the sample after corrosion were tested. The results showed that the surface of the copper mesh still maintained hydrophobicity. There was almost no loss of WCR, which remained at $5.26 \mathrm{~g} \mathrm{~cm}^{-2} \mathrm{~h}^{-1}$. It indicates that the water collection equipment made of the graphenebased polymer has high corrosion resistance. Zhou et al. ${ }^{108}$ prepared an alternate nanoscale hydrophobic-hydrophilic surface and Janus copper foam system (HB-HL + JCF) for fog collection by hydrothermal method and photocatalytic reaction. $\mathrm{ZnO}$ nanorods were uniformly grown into nanowires on $\mathrm{Cu}(\mathrm{OH})_{2}$ by the hydrothermal method and then some PDMS with low concentration was grafted onto $\mathrm{ZnO}$ nanorods under ultraviolet light. The layered structure showed enhanced fog collection ability. In order to test the weather resistance of HB-HL + JCF, it was subjected to multiple heating and cooling cycles and longterm ultraviolet irradiation. HB-HL + JCF was placed at a high temperature of $120{ }^{\circ} \mathrm{C}$ for 5 minutes, which was then immediately immersed in water at $20{ }^{\circ} \mathrm{C}$ for 2 minutes. After 10 heating and cooling cycles, it was found that the amount of water collected and the contact angle changed little. Because of the covalent bonds between the zinc atoms, oxygen atoms, and silicon atoms, the surface chemical stability could be maintained. After placing the HB-HL + JCF hydrophobic layer under an ultraviolet lamp for 36 hours, it was found that the water contact angle was reduced by $10^{\circ}$ and the mass of the collected water was reduced by $8.15 \%$. It showed that HB-HL + JCF still maintained a high WCA and WCR after being exposed to ultraviolet rays for a long time, showing excellent UV resistance.

In general, the use of a coating materials with good chemical stability is helpful in enhancing the corrosion resistance and weather resistance of the fog-collection surface. In addition, during the chemical etching or deposition process, it is necessary to ensure that the coating completely covers the substrate to reduce the possibility of the substrate being exposed to air.

\subsection{Antibacterial}

When the water collection equipment is applied in a humid environment for a long time, it may cause microorganisms to 
a
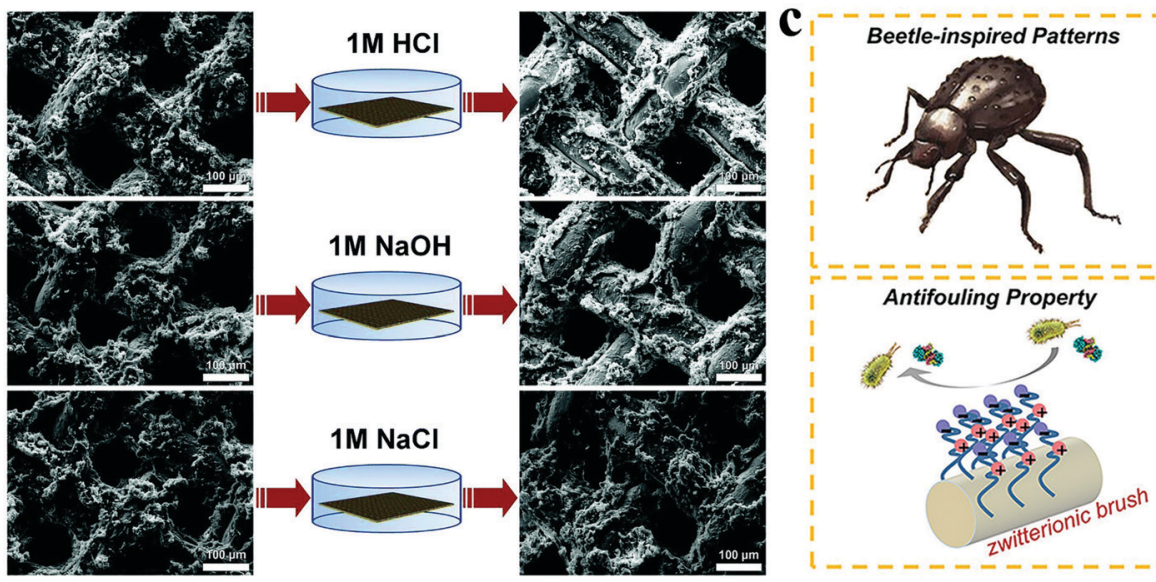

b
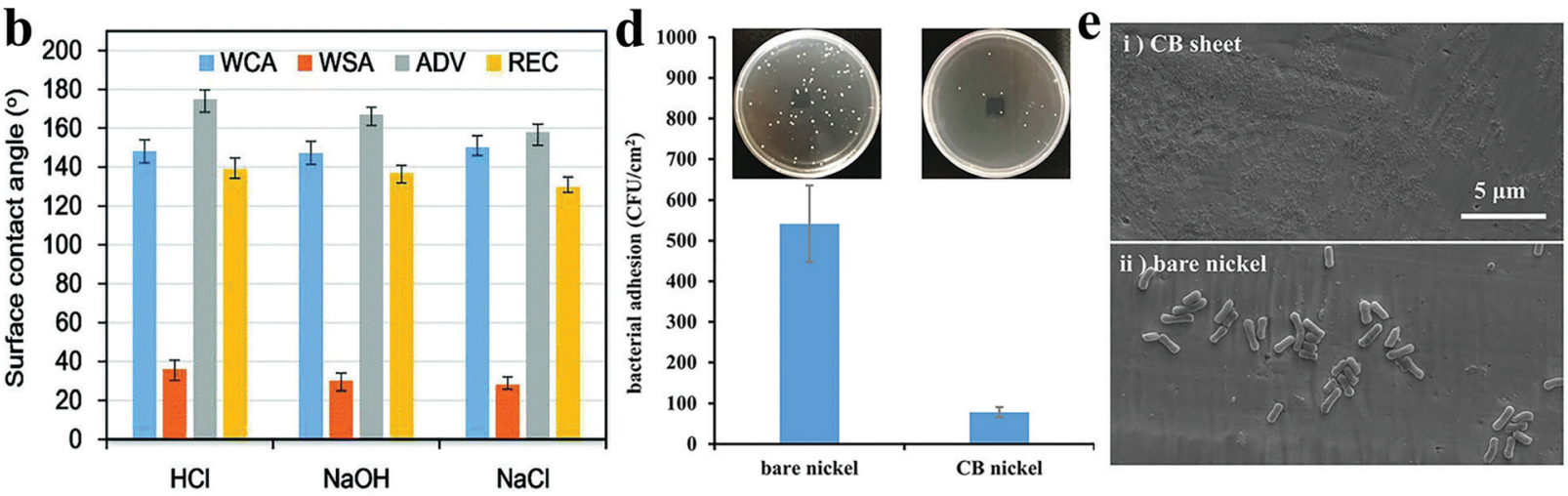

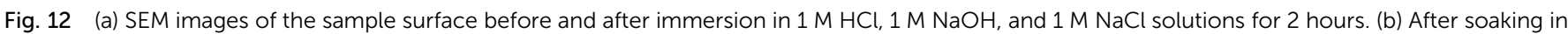

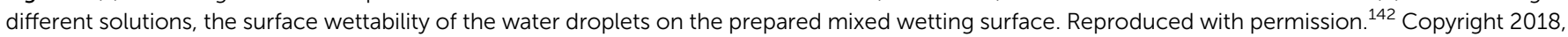

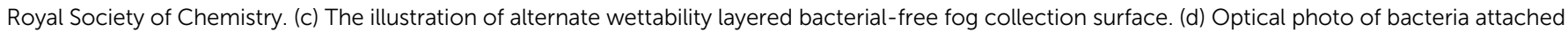

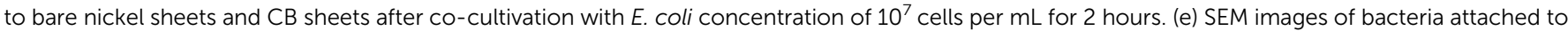

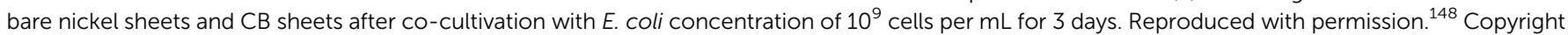
2019, American Chemical Society.

adhere and multiply on the surface of the water collection equipment, which can seriously affect the safety of water. ${ }^{143,144}$ So far, various antibacterial strategies with different mechanisms have been proposed. ${ }^{145,146}$ Studies have shown that superhydrophilic zwitterionic coating is a material that can effectively inhibit bacterial adhesion. ${ }^{147}$ The water layer is tightly bound to the superhydrophilic surface, thereby preventing bacteria from directly interacting with the surface and rapidly proliferating. Wen $e t \mathrm{al}^{148}$ proposed an alternate wettability layered fog collection interface based on antibacterial needle array ( $\mathrm{ABN}$ ) and desert beetle (Fig. 12c). Among them, ABN was coated with zwitterionic carboxybetaine (CB) brushes, which helped to efficiently capture fog droplets. The CB layer on the needle could promote the continuous capture of mist droplets and prevent bacterial contamination. The collected water droplets quickly separated from the surface of the hydrophobic sheet under the action of gravity. By changing the surface inclination angle and the number of CB needles, the WCR could be adjusted. When the inclination angle was fixed at $60^{\circ}$, ABN exhibited the highest WCR. In addition, as the number of $\mathrm{CB}$ needles gradually increased, the specific surface area of the water collection surface also increased, resulting in higher WCR. In order to test the antibacterial properties of the $\mathrm{CB}$ coating, bacterial adhesion tests were performed on bare nickel sheets and nickel sheets coated with CB. At an E. coli concentration of $10^{7}$ cells per $\mathrm{mL}$, bare nickel sheets and CB sheets were incubated for 2 hours. Optical photographs showed that approximately 542 bacteria adhered to the bare nickel plate (Fig. 12d). Correspondingly, approximately 78 bacteria adhered to the CB plate. At the E. coli concentration of $10^{9}$ cells per $\mathrm{mL}$, the bare nickel sheet and the CB sheet were co-cultured for 3 days. The SEM images showed no obvious dirt on the CB plate and the exposed nickel sheet was seriously contaminated by bacteria (Fig. 12e). The above results showed that the CB coating could prevent bacteria from directly contacting the surface of the substrate due to its strong hydration ability, thus exhibiting excellent antibacterial ability. The technology provides guidance for the future preparation of anti-bacterial water collection equipment.

In general, to improve the sustainability of the water collection surface, the design of structure, the choice of materials, and the preparation process must be considered. First of all, the armored structure is the most promising way to improve the wear resistance of water collection surfaces. Although there is little literature in this field, it is worth trying. Second, choosing right coating materials is 
a direct and effective way to improve the chemical stability and antibacterial properties. Finally, the preparation process is often overlooked. However, in electrochemical corrosion, chemical etching, spraying, and other processes, different parameters determine the density and thickness of the coating, which have a great impact on the performance of the water collection surface.

\section{Conclusion and outlook}

With rapid population growth and economic development, water shortage has become a worldwide problem. Therefore, it is imperative to develop new ways for obtaining fresh water. Inspired by nature, bioinspired water harvesting materials have become a research hot topic in recent years. To improve the water collection efficiency and performance, excellent microstructure design and sustainability are essential.

In this review, we focus on the microstructure design and sustainability of bioinspired water harvesting surfaces. According to different mechanisms, the water collection surface is roughly divided into three categories: one-dimensional structure, twodimensional structure, and composite structure. Among them, wettability is the key in the droplet capture process. Droplets deposit faster on hydrophilic surfaces than on hydrophobic surfaces but hydrophobic surfaces are more conducive for the continuous refreshing of the water collection process. Laplace pressure, chemical gradient force, capillary wetting force, and gravity are several driving forces that water droplets may receive during transportation. The composite structure combines a variety of mechanisms, exhibiting faster water transmission speed and WCR than the one-dimensional structure and the twodimensional structure. This is the direction that should be paid attention to in the future to improve the WCR. In addition, we conclude that the sustainability of the water collection surface is mainly manifested in the mechanical stability, chemical stability, and antibacterial properties. Herein, we have provided a summary of some previous works, discussing how to improve the durability and safety. The multi-scale hierarchical structure may help the water collection surface to improve the robustness. The use of chemically stable and bacteria-resistant materials as the coating can effectively resist acid-base corrosion, high temperature, strong ultraviolet rays, and bacterial contamination.

At present, the research on water collection surfaces is still at the laboratory stage. To move towards large-scale industrial applications, several issues need to be resolved. On the one hand, it is necessary to explore more water collection mechanisms of natural organisms and to strengthen the theoretical research, which is conducive for the development of water collection materials that are different from existing mechanisms. On the other hand, the performance of water harvesting is affected by many factors so that it should be explored in more depth to provide references for researchers to improve the water collection efficiency. Moreover, the water collection rate test is often conducted in a closed environment where the humidifier provides more than $80 \%$ humidity, which is different from air humidity in arid areas. Different standards lead to different water collection rates; consequently, uniform testing standards have become particularly important. In addition, while trying to improve the efficiency, the tolerance of the collection surface in harsh environments should not be ignored. It is significant to improve the weather resistance and antibacterial performance of water-collecting materials to prolong the service life of the equipment. Finally, the commercialization of bioinspired water collection materials is very important. At present, due to the complex preparation process and the lack of production lines, the size and price of commercial water collection surfaces limit large-scale industrial applications, which prevent it from being promoted worldwide. Therefore, purchasing raw materials uniformly, optimizing the preparation process, and establishing standard production lines are the most critical steps for producing water harvesting materials so as to move towards real life applications.

To sum up, the ultimate purpose of water-collecting materials is to increase the utilization rate of freshwater resources. Nowadays, they show great potential in microfluidics, biology, medicine, and other fields. In the future, there are reasons to believe that bioinspired water harvesting materials will develop in the direction of lightness, large size, superior performance, and cheapness, which can bring great convenience to our lives.

\section{Conflicts of interest}

The authors declare no conflict of interest.

\section{Acknowledgements}

This work was financially supported by the National Natural Science Foundation of China (No. 51675513 and 51735013).

\section{Notes and references}

1 N. W. Arnell, D. P. van Vuuren and M. Isaac, Global Environ. Change, 2011, 21, 592-603.

2 T. Oki and S. Kanae, Science, 2006, 313, 1068-1072.

3 C. Pahl-Wostl, M. Craps, A. Dewulf, E. Mostert, D. Tabara and T. Taillieu, Ecol. Soc., 2007, 12, 5.

4 S. Piao, P. Ciais, Y. Huang, Z. Shen, S. Peng, J. Li, L. Zhou, H. Liu, Y. Ma and Y. Ding, Nature, 2010, 467, 43.

5 S. L. Postel, G. C. Daily and P. R. Ehrlich, Science, 1996, 271, 785-788.

6 C. J. Vörösmarty, P. Green, J. Salisbury and R. B. Lammers, Science, 2000, 289, 284-288.

7 J. Alcamo, M. Flörke and M. Märker, Hydrol. Sci. J., 2007, 52, 247-275.

8 I. Aymerich, L. Rieger, R. Sobhani, D. Rosso and L. Corominas, Water Res., 2015, 81, 113-123.

9 P. K. Cornejo, J. Becker, K. Pagilla, W. Mo, Q. Zhang, J. R. Mihelcic, K. Chandran, B. Sturm, D. Yeh and D. Rosso, Water Environ. Res., 2018, 91, 45-53. 
10 S. M. Hanna, M. J. Thompson, M. F. Dahab, R. E. Williams and B. I. Dvorak, Water Environ. Res., 2018, 90, 738-747.

11 K. J. An, Y. F. Lam, S. Hao, T. E. Morakinyo and H. Furumai, Water Res., 2015, 86, 116-121.

12 D. Kim, J. D. Bowen and D. Kinnear, Water Environ. Res., 2015, 87, 1955-1969.

13 R. Li, Y. Shi, M. Wu, S. Hong and P. Wang, Nano Energy, 2020, 67, 104255.

14 C. Prouty, S. Mohebbi and Q. Zhang, Water Res., 2018, 137, 107-119.

15 Y. Tu, R. Wang, Y. Zhang and J. Wang, Joule, 2018, 2, 1452-1475.

16 R. Tu and Y. Hwang, Energy, 2020, 201, 117630.

17 M. M. Mekonnen and A. Y. Hoekstra, Sci. Adv., 2016, 2, e1500323.

18 M. Elimelech and W. A. Phillip, Science, 2011, 333, 712-717.

19 H. M. Qiblawey and F. Banat, Desalination, 2008, 220, 633-644.

20 S. Parekh, M. Farid, J. Selman and S. Al-Hallaj, Desalination, 2004, 160, 167-186.

21 R. V. Linares, Z. Li, S. Sarp, S. S. Bucs, G. Amy and J. S. Vrouwenvelder, Water Res., 2014, 66, 122-139.

22 K. Lutchmiah, A. Verliefde, K. Roest, L. C. Rietveld and E. Cornelissen, Water Res., 2014, 58, 179-197.

23 H. Kim, S. Yang, S. R. Rao, S. Narayanan, E. A. Kapustin, H. Furukawa, A. S. Umans, O. M. Yaghi and E. N. Wang, Science, 2017, 356, 430.

24 M. Fessehaye, S. A. Abdul-Wahab, M. J. Savage, T. Kohler, T. Gherezghiher and H. Hurni, Renewable Sustainable Energy Rev., 2014, 29, 52-62.

25 Y. Zheng, H. Bai, Z. Huang, X. Tian, F.-Q. Nie, Y. Zhao, J. Zhai and L. Jiang, Nature, 2010, 463, 640.

26 V. Sharma, D. Orejon, Y. Takata, V. Krishnan and S. Harish, ACS Sustainable Chem. Eng., 2018, 6, 6981-6993.

27 V. Sharma, M. Sharma, S. Kumar and V. Krishnan, Flora, 2016, 224, 59-65.

28 V. Sharma, S. Kumar, K. L. Reddy, A. Bahuguna and V. Krishnan, J. Mol. Eng. Mater., 2016, 04, 1640006.

29 A. R. Parker and C. R. Lawrence, Nature, 2001, 414, 33.

30 P. Gandhidasan and H. I. Abualhamayel, Water Environ. J., 2007, 21, 19-25.

31 G. A. Al-hassan, Water Resour. Manag., 2009, 23, 2805-2813.

32 H. Cho, B. Park, M. Kim, S. Lee and W. Hwang, J. Mater. Chem. A, 2017, 5, 25328-25337.

33 H. Luo, Y. Lu, S. Yin, S. Huang, J. Song, F. Chen, F. Chen, C. J. Carmalt and I. P. Parkin, J. Mater. Chem. A, 2018, 6, 5635-5643.

34 S. Zhang, J. Huang, Z. Chen, S. Yang and Y. Lai, J. Mater. Chem. A, 2019, 7, 38-63.

35 D. Li, Z. Wang, D. Wu, G. Han and Z. Guo, Nanoscale, 2019, 11, 11774-11781.

36 Y. Liu, N. Yang, X. Li, J. Li, W. Pei, Y. Xu, Y. Hou and Y. Zheng, Small, 2020, 16, 1901819.

37 X. Wang, J. Zeng, X. Yu and Y. Zhang, J. Mater. Chem. A, 2019, 7, 5426-5433.

38 J. Lu, C.-V. Ngo, S. C. Singh, J. Yang, W. Xin, Z. Yu and C. Guo, Langmuir, 2019, 35, 3562-3567.
39 Y. Hou, Y. Shang, M. Yu, C. Feng, H. Yu and S. Yao, ACS Nano, 2018, 12, 11022-11030.

40 Y. Dou, D. Tian, Z. Sun, Q. Liu, N. Zhang, J. H. Kim, L. Jiang and S. X. Dou, ACS Nano, 2017, 11, 2477-2485.

41 Z. Sun, T. Liao, W. Li, Y. Dou, K. Liu, L. Jiang, S.-W. Kim, J. Ho Kim and S. Xue Dou, NPG Asia Mater., 2015, 7, e232-e232.

42 Z. Sun, T. Liao, K. Liu, L. Jiang, J. H. Kim and S. X. Dou, Small, 2014, 10, 3001-3006.

43 Y. Zhang, J. Mei, C. Yan, T. Liao, J. Bell and Z. Sun, Adv. Mater., 2020, 32, 1902806.

44 Y. Chen, D. Li, T. Wang and Y. Zheng, Sci. Rep., 2016, 6, 19978.

45 J. Ju, H. Bai, Y. Zheng, T. Zhao, R. Fang and L. Jiang, Nat. Commun., 2012, 3, 1247.

46 H. Bai, L. Wang, J. Ju, R. Sun, Y. Zheng and L. Jiang, Adv. Mater., 2014, 26, 5025-5030.

47 Y. Zhang, D. Ge and S. Yang, J. Colloid Interface Sci., 2014, 423, 101-107.

48 X. Jing and Z. Guo, ACS Appl. Mater. Interfaces, 2019, 11, 35949-35958.

49 T. Young, Philos. Trans. R. Soc. London, 1805, 95, 65-87.

50 R. N. Wenzel, Ind. Eng. Chem. Res., 1936, 28, 988-994.

51 A. Cassie and S. Baxter, Trans. Faraday Soc., 1944, 40, 546-551.

52 C. Andrieu, D. A. Beysens, V. S. Nikolayev and Y. Pomeau, J. Fluid Mech., 2002, 453, 427-438.

53 H. Zhao and D. Beysens, Langmuir, 1995, 11, 627-634.

54 É. Lorenceau and D. Quéré, J. Fluid Mech., 2004, 510, 29-45.

55 M. K. Chaudhury and G. M. Whitesides, Science, 1992, 256, 1539-1541.

56 T. Nørgaard, M. Ebner and M. Dacke, PLoS One, 2012, 7, e34603.

57 A. Roth-Nebelsick, M. Ebner, T. Miranda, V. Gottschalk, D. Voigt, S. Gorb, T. Stegmaier, J. Sarsour, M. Linke and W. Konrad, J. R. Soc., Interface, 2012, 9, 1965-1974.

58 L. Zhai, M. C. Berg, F. C. Cebeci, Y. Kim, J. M. Milwid, M. F. Rubner and R. E. Cohen, Nano Lett., 2006, 6, 1213-1217.

59 R. Garrod, L. Harris, W. Schofield, J. McGettrick, L. Ward, D. Teare and J. Badyal, Langmuir, 2007, 23, 689-693.

60 C. Dorrer and J. R. Rühe, Langmuir, 2008, 24, 6154-6158.

61 N. Thakur, A. S. Ranganath, K. Agarwal and A. Baji, Macromol. Mater. Eng., 2017, 302, 1700124.

62 Y. Liu, N. Yang, C. Gao, X. Li, Z. Guo, Y. Hou and Y. Zheng, ACS Appl. Mater. Interfaces, 2020, 12, 28876-28884.

63 H. Bai, X. Tian, Y. Zheng, J. Ju, Y. Zhao and L. Jiang, Adv. Mater., 2010, 22, 5521-5525.

64 H. Bai, J. Ju, R. Sun, Y. Chen, Y. Zheng and L. Jiang, Adv. Mater., 2011, 23, 3708-3711.

65 Y. Hou, Y. Chen, Y. Xue, Y. Zheng and L. Jiang, Langmuir, 2012, 28, 4737-4743.

66 Z. Huang, Y. Chen, Y. Zheng and L. Jiang, Soft Matter, 2011, 7, 9468-9473.

67 X. Tian, Y. Chen, Y. Zheng, H. Bai and L. Jiang, Adv. Mater., 2011, 23, 5486-5491. 
68 S. Wang, S. Feng, Y. Hou and Y. Zheng, Macromol. Rapid Commun., 2015, 36, 459-464.

69 Y. Chen, L. Wang, Y. Xue, L. Jiang and Y. Zheng, Sci. Rep., 2013, 3, 2927.

70 H. Venkatesan, J. Chen, H. Liu, W. Liu and J. Hu, Adv. Funct. Mater., 2020, 30, 2002437.

71 S. J. Lee, N. Ha and H. Kim, ACS Sustainable Chem. Eng., 2019, 7, 10561-10569.

72 S. Yi, J. Wang, Z. Chen, B. Liu, L. Ren, L. Liang and L. Jiang, Adv. Mater. Technol., 2019, 4, 1900727.

73 J. Wang, S. Yi, Z. Yang, Y. Chen, L. Jiang and C.-P. Wong, ACS Appl. Mater. Interfaces, 2020, 12, 21080-21087.

74 M. Cao, J. Ju, K. Li, S. Dou, K. Liu and L. Jiang, Adv. Funct. Mater., 2014, 24, 3235-3240.

75 X. Heng, M. Xiang, Z. Lu and C. Luo, ACS Appl. Mater. Interfaces, 2014, 6, 8032-8041.

76 J. Ju, K. Xiao, X. Yao, H. Bai and L. Jiang, Adv. Mater., 2013, 25, 5937-5942.

77 J. Zou, P. F. Wang, T. R. Zhang, X. Fu and X. Ruan, Phys. Fluids, 2011, 23, 044101.

78 J. Ju, X. Yao, S. Yang, L. Wang, R. Sun, Y. He and L. Jiang, Adv. Funct. Mater., 2014, 24, 6933-6938.

79 X. Li, Y. Yang, L. Liu, Y. Chen, M. Chu, H. Sun, W. Shan and Y. Chen, Adv. Mater. Interfaces, 2020, 7, 1901752.

80 L. Zhong, H. Zhu, Y. Wu and Z. Guo, J. Colloid Interface Sci., 2018, 525, 234-242.

81 H. Zhu, F. Yang, J. Li and Z. Guo, Chem. Commun., 2016, 52, 12415-12417.

82 K.-C. Park, P. Kim, A. Grinthal, N. He, D. Fox, J. C. Weaver and J. Aizenberg, Nature, 2016, 531, 78-82.

83 J. Lei and Z. Guo, Nanoscale, 2020, 12, 6921-6936.

84 M. Wang, Q. Liu, H. Zhang, C. Wang, L. Wang, B. Xiang, Y. Fan, C. F. Guo and S. Ruan, ACS Appl. Mater. Interfaces, 2017, 9, 29248-29254.

85 Z. Wang, A. Owais, C. Neto, J.-M. Pereira and Y. Gan, Adv. Mater. Interfaces, 2020, 2000520, DOI: 10.1002/admi. 202000520.

86 S. Wang, C. Wang, Z. Peng and S. Chen, J. Phys. Chem. C, 2019, 123, 1798-1805.

87 M. Liu, Y. Yao, Y. Yang, Z. Peng and S. Chen, J. Phys. Chem. $C, 2019,123,12736-12743$.

88 S. Wang, Z. Peng, J. Li, Y. Yang, C. Wang and S. Chen, Comput. Mater. Sci., 2020, 175, 109616.

89 Y.-Y. Song, Y. Liu, H.-B. Jiang, S.-Y. Li, C. Kaya, T. Stegmaier, Z.-W. Han and L.-Q. Ren, Nanoscale, 2018, 10, 3813-3822.

90 M.-G. Medici, A. Mongruel, L. Royon and D. Beysens, Phys. Rev. E: Stat., Nonlinear, Soft Matter Phys., 2014, 90, 062403.

91 J. L. Viovy, D. Beysens and C. M. Knobler, Phys. Rev. A, 1988, 37, 4965-4970.

92 Y. Wang, X. Wang, C. Lai, H. Hu, Y. Kong, B. Fei and J. H. Xin, ACS Appl. Mater. Interfaces, 2016, 8, 2950-2960.

93 X. Liu and P. Cheng, Int. J. Heat Mass Transfer, 2015, 83, 842-849.

94 K. K. Varanasi, M. Hsu, N. Bhate, W. Yang and T. Deng, Appl. Phys. Lett., 2009, 95, 094101.
95 X. Dai, N. Sun, S. O. Nielsen, B. B. Stogin, J. Wang, S. Yang and T.-S. Wong, Sci. Adv., 2018, 4, eaaq0919.

96 W. Chen and Z. Guo, Nanoscale, 2019, 11, 15448-15463.

97 J. W. Rose, Proc. Inst. Mech. Eng., Part A, 2002, 216, 115-128.

98 Y. Zhao, D. J. Preston, Z. Lu, L. Zhang, J. Queeney and E. N. Wang, Int. J. Heat Mass Transfer, 2018, 119, 931-938.

99 X. Ji, D. Zhou, C. Dai and J. Xu, Int. J. Heat Mass Transfer, 2019, 132, 52-67.

100 J. B. Boreyko and C.-H. Chen, Phys. Rev. Lett., 2009, 103, 184501.

101 B. S. Sikarwar, N. K. Battoo, S. Khandekar and K. Muralidhar, J. Heat Transfer, 2010, 133, 021501.

102 J. Feng, Z. Qin and S. Yao, Langmuir, 2012, 28, 6067-6075.

103 N. Miljkovic, R. Enright, Y. Nam, K. Lopez, N. Dou, J. Sack and E. N. Wang, Nano Lett., 2013, 13, 179-187.

104 K. Yin, H. Du, X. Dong, C. Wang, J.-A. Duan and J. He, Nanoscale, 2017, 9, 14620-14626.

105 J. Ai and Z. Guo, Chem. Commun., 2019, 55, 10820-10843. 106 X. Wang, J. Zeng, X. Yu, C. Liang and Y. Zhang, RSC Adv., 2020, 10, 282-288.

107 P. Zhang, B. Peng, J. Wang and L. Jiang, Adv. Funct. Mater., 2019, 29, 1904535.

108 H. Zhou, X. Jing and Z. Guo, J. Colloid Interface Sci., 2020, 561, 730-740.

109 H. Zhou and Z. Guo, J. Mater. Chem. A, 2019, 7, 12921-12950.

110 K. Yin, S. Yang, X. Dong, D. Chu, J.-A. Duan and J. He, ACS Appl. Mater. Interfaces, 2018, 10, 31433-31440.

111 Y. Zhao, C. Yu, H. Lan, M. Cao and L. Jiang, Adv. Funct. Mater., 2017, 27, 1701466.

112 H.-C. Yang, Y. Xie, J. Hou, A. K. Cheetham, V. Chen and S. B. Darling, Adv. Mater., 2018, 30, 1801495.

113 X. Tian, J. Li and X. Wang, Soft Matter, 2012, 8, 2633-2637.

114 Y.-P. An, J. Yang, H.-C. Yang, M.-B. Wu and Z.-K. Xu, ACS Appl. Mater. Interfaces, 2018, 10, 9832-9840.

115 Z. Wang, Y. Wang and G. Liu, Angew. Chem., Int. Ed., 2016, 55, 1291-1294.

116 X. Yang, Z. Wang and L. Shao, J. Membr. Sci., 2018, 549, 67-74.

117 H. Wang, H. Zhou, H. Niu, J. Zhang, Y. Du and T. Lin, Adv. Mater. Interfaces, 2015, 2, 1400506.

118 H.-C. Yang, W. Zhong, J. Hou, V. Chen and Z.-K. Xu, J. Membr. Sci., 2017, 523, 1-7.

119 A. Alkhudhiri, N. Darwish and N. Hilal, Desalination, 2012, 287, 2-18.

120 P. Tian, X. Gao, G. Wen, L. Zhong, Z. Wang and Z. Guo, J. Colloid Interface Sci., 2018, 532, 517-526.

121 H. Cheng, F. Zhao, J. Xue, G. Shi, L. Jiang and L. Qu, ACS Nano, 2016, 10, 9529-9535.

122 M. Cao, J. Xiao, C. Yu, K. Li and L. Jiang, Small, 2015, 11, 4379-4384.

123 H. Zhou, M. Zhang, C. Li, C. Gao and Y. Zheng, Small, 2018, 14, 1801335.

124 L. Zhong, J. Feng and Z. Guo, J. Mater. Chem. A, 2019, 7, 8405-8413. 
125 C. Li, Y. Liu, C. Gao, X. Li, Y. Xing and Y. Zheng, ACS Appl. Mater. Interfaces, 2019, 11, 4507-4513.

126 R. Hu, N. Wang, L. Hou, Z. Cui, J. Liu, D. Li, Q. Li, H. Zhang and Y. Zhao, J. Mater. Chem. A, 2019, 7, 124-132.

127 V. Sharma, R. Balaji and V. Krishnan, Biomimetics, 2018, 3, 7.

128 H. Chen, T. Ran, Y. Gan, J. Zhou, Y. Zhang, L. Zhang, D. Zhang and L. Jiang, Nat. Mater., 2018, 17, 935-942.

129 T. Verho, C. Bower, P. Andrew, S. Franssila, O. Ikkala and R. H. A. Ras, Adv. Mater., 2011, 23, 673-678.

130 A. Milionis, E. Loth and I. S. Bayer, Adv. Colloid Interface Sci., 2016, 229, 57-79.

131 X. Deng, L. Mammen, H.-J. Butt and D. Vollmer, Science, 2012, 335, 67.

132 W. Jiang, H. Liu, L. Wang, S. Zhu, L. Yin, Y. Shi, B. Chen, Y. Ding and N. An, Thin Solid Films, 2014, 562, 383-388.

133 Y. Li, S. Chen, M. Wu and J. Sun, Adv. Mater., 2014, 26, 3344-3348.

134 T. Maitra, C. Antonini, M. Auf der Mauer, C. Stamatopoulos, M. K. Tiwari and D. Poulikakos, Nanoscale, 2014, 6, 8710-8719.

135 Y. Liu, Z. Liu, Y. Liu, H. Hu, Y. Li, P. Yan, B. Yu and F. Zhou, Small, 2015, 11, 426-431.

136 H. Wang, Z. Liu, X. Zhang, C. Lv, R. Yuan, Y. Zhu, L. Mu and J. Zhu, Adv. Mater. Interfaces, 2016, 3, 1600040.

137 X. Zhang, Z. Liu, Y. Li, Y. Cui, H. Wang and J. Wang, Chem. Eng. J., 2020, 392, 123741.
138 A. C. C. Esteves, Y. Luo, M. W. P. van de Put, C. C. M. Carcouët and G. de With, Adv. Funct. Mater., 2014, 24, 986-992.

139 Y. Lu, S. Sathasivam, J. Song, C. R. Crick, C. J. Carmalt and I. P. Parkin, Science, 2015, 347, 1132.

140 W. S. Y. Wong, Z. H. Stachurski, D. R. Nisbet and A. Tricoli, ACS Appl. Mater. Interfaces, 2016, 8, 13615-13623.

141 D. Wang, Q. Sun, M. J. Hokkanen, C. Zhang, F.-Y. Lin, Q. Liu, S.-P. Zhu, T. Zhou, Q. Chang, B. He, Q. Zhou, L. Chen, Z. Wang, R. H. A. Ras and X. Deng, Nature, 2020, 582, 55-59.

142 Y.-y. Song, Y. Liu, H.-b. Jiang, S.-y. Li, C. Kaya, T. Stegmaier, Z.-w. Han and L.-q. Ren, Nanoscale, 2018, 10, 16127-16137.

143 S. Naderizadeh, S. Dante, P. Picone, M. Di Carlo, R. Carzino, A. Athanassiou and I. S. Bayer, J. Colloid Interface Sci., 2020, 574, 20-32.

144 H. Fan and Z. Guo, Biomater. Sci., 2020, 8, 1502-1535.

145 T. Wei, Z. Tang, Q. Yu and H. Chen, ACS Appl. Mater. Interfaces, 2017, 9, 37511-37523.

146 P. Zhang, L. Lin, D. Zang, X. Guo and M. Liu, Small, 2017, 13, 1503334.

147 G. Cheng, Z. Zhang, S. Chen, J. D. Bryers and S. Jiang, Biomaterials, 2007, 28, 4192-4199.

148 C. Wen, H. Guo, H. Bai, T. Xu, M. Liu, J. Yang, Y. Zhu, W. Zhao, J. Zhang, M. Cao and L. Zhang, ACS Appl. Mater. Interfaces, 2019, 11, 34330-34337. 